\title{
Biophysical basis of cellular multi-specificity encoded in a model molecular switch
}

\author{
Tina Perica ${ }^{1,2,3,10}$, Christopher J. P. Mathy $y^{1,2,4,10}$, Jiewei Xu ${ }^{2,5,6}$, Gwendolyn M. Jang ${ }^{2,5,6}$, Yang Zhang ${ }^{1,2}$, Robyn Kaake ${ }^{2,5,6}$, \\ Noah Ollikainen ${ }^{1,2,7}$, Hannes Braberg ${ }^{2,5}$, Danielle L. Swaney ${ }^{2,5,6}$, Mark J. S. Kelly ${ }^{8}$, Nevan J. Krogan ${ }^{2,5,6, \mathbb{}}$, and Tanja \\ Kortemme $\mathbf{e}^{1,2,4,7,9, \mathbb{}}$
${ }^{1}$ Department of Bioengineering and Therapeutic Sciences, University of California San Francisco, San Francisco, CA, USA
${ }^{2}$ Quantitative Biosciences Institute, University of California San Francisco, San Francisco, CA, USA ${ }^{3}$ European Molecular Biology Laboratory, European Bioinformatics Institute (EMBL-EBI), Hinxton, Cambridge, UK
${ }^{5}$ Department of Cellular and Molecular Pharmacology, University of California San Francisco, San Francisco, CA, USA ${ }^{6}$ The J. David Gladstone Institutes, San Francisco, CA, USA
${ }^{7}$ Graduate Program in Bioinformatics, University of California San Francisco, San Francisco, California, USA
${ }^{8}$ Department of Pharmaceutical Chemistry, University of California, San Francisco, San Francisco, CA, USA ${ }^{9}$ Chan Zuckerberg Biohub, San Francisco, USA
${ }^{10}$ These authors contributed equally \\ ${ }^{4}$ The UC Berkeley-UCSF Graduate Program in Bioengineering, University of California San Francisco, San Francisco, CA, USA
}

\begin{abstract}
Molecular switches are central to signal transduction in protein interaction networks. One switch protein can independently regulate distinct cellular processes, but the molecular mechanisms enabling this functional multi-specificity remain unclear. Here we integrate system-scale cellular and biophysical measurements to study how a paradigm switch, the small GTPase Ran/Gsp1, achieves its functional multi-specificity. We make 56 targeted point mutations to individual interactions of Ran/Gsp1 and show through quantitative, systematic genetic and physical interaction mapping that Ran/Gsp1 interface perturbations have widespread cellular consequences that cluster by biological processes but, unexpectedly, not by the targeted interactions. Instead, the cellular consequences of the interface mutations group by their biophysical effects on kinetic parameters of the GTPase switch cycle, and cycle kinetics are allosterically tuned by distal interface mutations. We propose that the functional multi-specificity of Ran/Gsp1 is encoded by a differential sensitivity of biological processes to different kinetic parameters of the Gsp1 switch cycle, and that Gsp1 partners binding to the sites of distal mutations act as allosteric regulators of the switch. Similar mechanisms may underlie biological regulation by other GTPases and biological switches. Finally, our integrative platform to determine the quantitative consequences of cellular perturbations may help explain the effects of disease mutations targeting central switches.
\end{abstract}

Ran | Gsp1 | GTPase | molecular switches | systems biology | thermodynamic coupling

Correspondence: kortemme@cgl.ucsf.edu nevan.krogan@ucsf.edu

Proteins perform their cellular functions within networks of interactions with many partners $(1,2)$. This complexity raises the fundamental question of functional specificity: How can different functions be controlled individually with the required precision and accuracy, when distinct cellular processes are interconnected and often even share common regulators? Moreover, in highly interconnected networks even a small perturbation targeting individual interactions, introduced by posttranslational modifications, point mutations, or drug binding, could be magnified through the network and have widespread cellular consequences. Protein mutations in disease are enriched in protein-protein interfaces $(3,4)$, but it is unclear whether the consequences of these mutations can be explained primarily by their effects on individ- ual interactions. Similarly, drug compounds are typically designed against specific targets but could affect cellular functions more broadly. Determining the extent and mechanism by which molecular perturbations affect interconnected biological processes requires an approach that quantifies effects on both the cellular network and on the molecular functions of the targeted protein (Fig. 1a).

To develop such an approach, we targeted a central molecular switch, a GTPase. GTPases belong to a class of common biological motifs, where a two-state switch is controlled by regulators with opposing functions $(6,7)$ (Fig. 1a). For GTPases, the two states of the switch are defined by the conformation of the GTPase in either the GTP- or GDPbound forms, and the interconversion between the two states is catalyzed by guanine nucleotide exchange factors (GEFs) and GTPase-activating proteins (GAPs) (Fig. 1b). Switch motifs are often multi-specific, defined here as regulating several different processes (8). This multi-specificity raises the question of how a single switch motif differentially controls diverse processes at the cellular level.

In this study, we sought to uncover the mechanistic basis of functional multi-specificity in the small GTPase Gsp1 (the $S$. cerevisiae homolog of human Ran, which shares $83 \%$ amino acid identity with Gsp1), which is a single molecular switch with one main GEF and one main GAP (9). Gsp1 regulates nucleocytoplasmic transport of proteins $(10,11)$ and RNA $(12,13)$, cell cycle progression (14), RNA processing (15) and nuclear envelope assembly (16). Gsp1/Ran forms direct physical interactions with a large number of partners, and high-resolution crystal structures of Gsp1/Ran in complex with 16 different binding partners are known (Fig. S1, Supplementary File 1 Table 1). We reasoned that by placing defined point mutations in Gsp1 interfaces with these partners, we could differentially perturb subsets of biological processes regulated by Gsp1. We then determined the functional consequences of these Gsp1 mutations on diverse biological processes in $S$. cerevisiae using quantitative genetic interaction mapping, measured changes to the physical interaction network using affinity purification mass spectrometry (AP-MS), and finally quantified molecular changes on the 
bioRxiv preprint doi: https://doi.org/10.1101/2020.01.04.893909; this version posted January 6, 2020. The copyright holder for this preprint (which was not certified by peer review) is the author/funder, who has granted bioRxiv a license to display the preprint in perpetuity. It is made available under aCC-BY-NC-ND 4.0 International license.
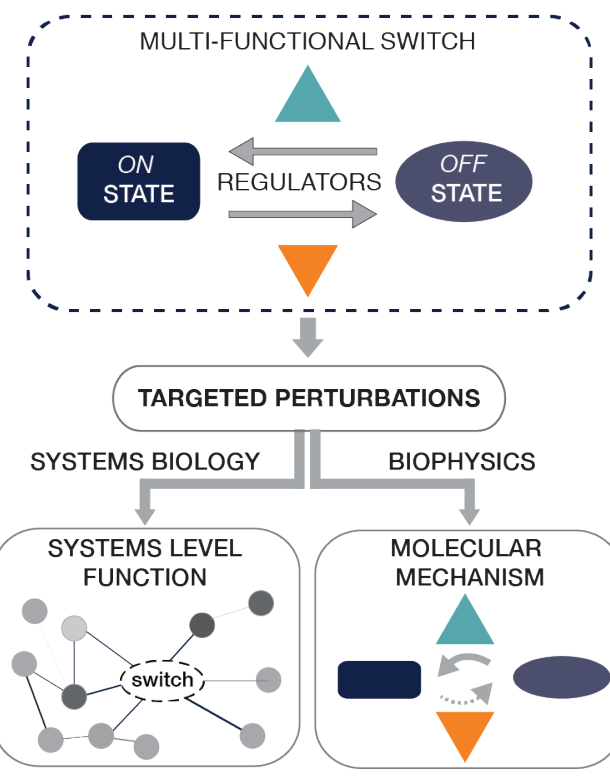

d
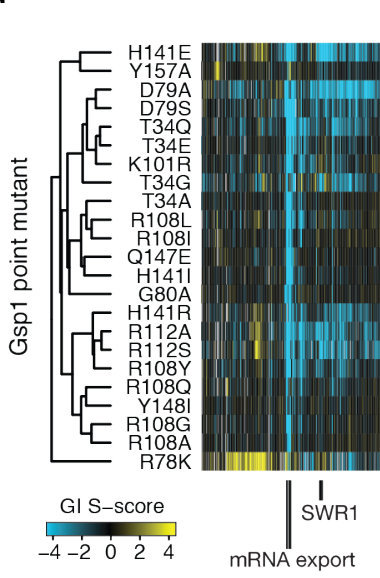

S. cerevisiae E-MAP deletion library $(n=1444)$

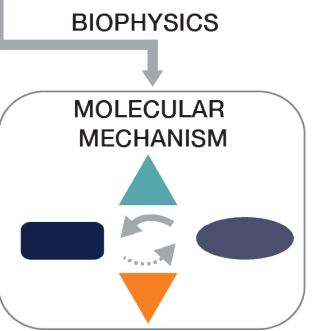

b
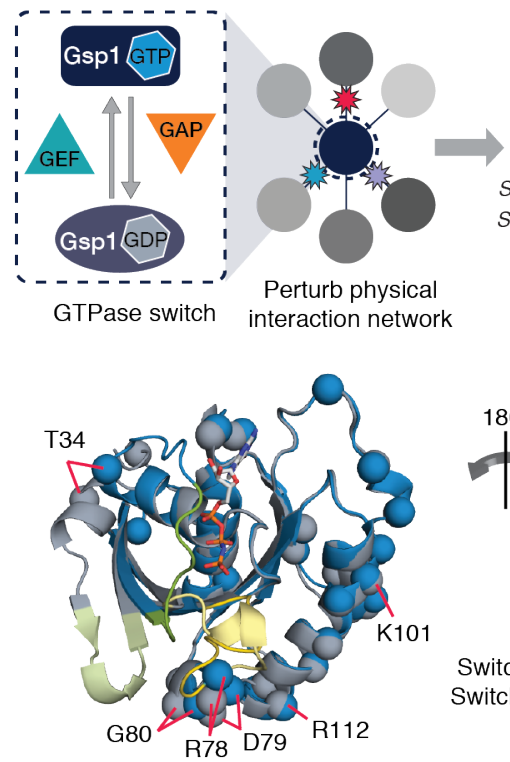

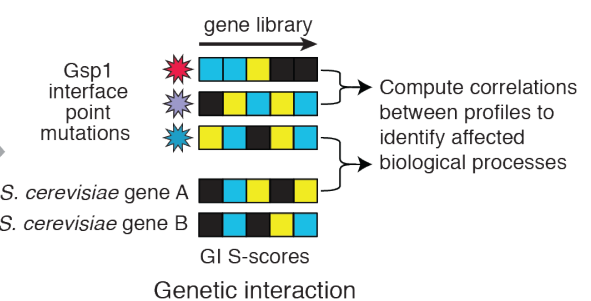

Genetic interaction profiling

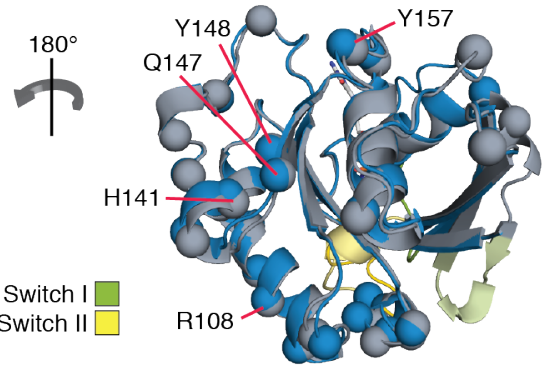

Dynein/Dynactin urNA Dynein/Dynactin urmylation Spindle assembly

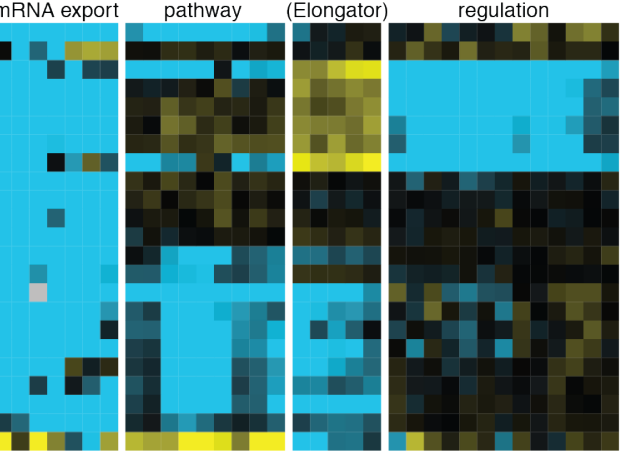

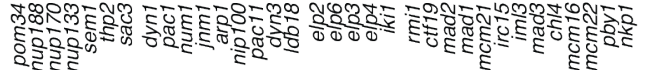

으ㅇㅡㅗ을 Nuclear por complex e

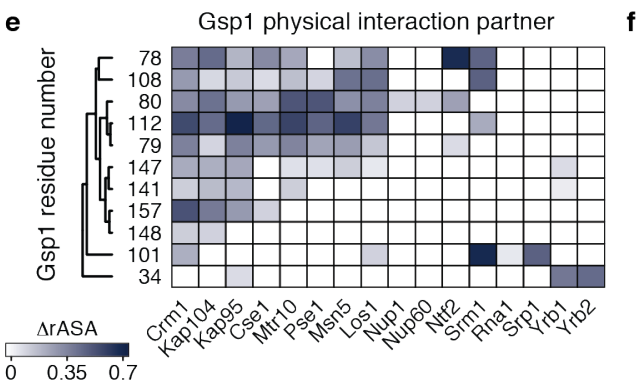

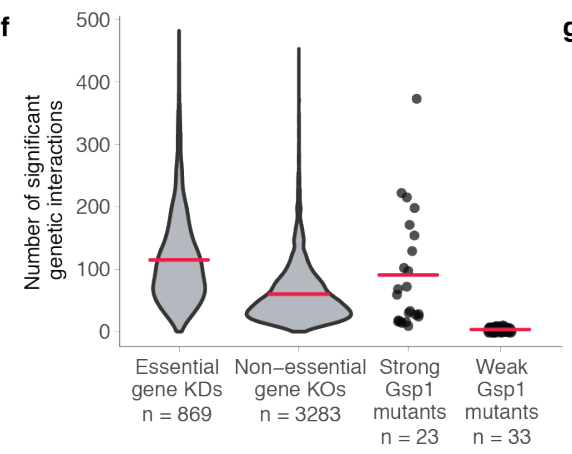

g

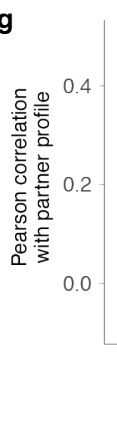

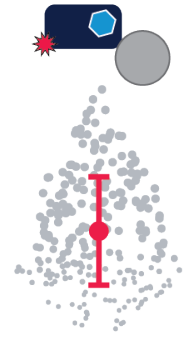
$\begin{array}{cc}\text { Strong mutant } & \text { Strong mutant } \\ \text { not in partner interface } & \text { in partner interface }\end{array}$ $n=240$

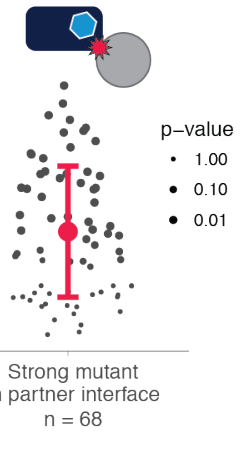

Fig. 1. Genetic interaction (GI) profiles of Gsp1 interface point mutants cluster by biological processes but not by targeted interfaces. a, Schematic summary of approach combining systems-level and biophysical measurements to characterize functional multi-specificity of a biological switch motif. b, Interface point mutations enable probing of the biological functions of the multi-specific GTPase switch Gsp1. c, Structures of Ran/Gsp1 in the GTP-bound (marine, PDB ID: 1ibr) and GDP-bound (gray, PDB ID: 3gj0) states. Mutated Gsp1 residues are shown as spheres. Switch loops I and II are shown in green and yellow, respectively. d, GI profiles of 23 Gsp1 mutants with nine or more significant Gls. Negative S-score (blue) represents synthetic sick/lethal Gls, positive S-score (yellow) represents suppressive/epistatic Gls. Mutants and genes are hierarchically clustered by Pearson correlation. e, Locations of mutated residues in structurally characterized interfaces. $\Delta$ rASA is the difference in accessible surface area of a residue upon binding, relative to an empirical maximum for the solvent accessible surface area of each amino acid residue type computed as in (5). $\mathbf{f}$, Distributions of significant (see Methods) Gls of Gsp1 point mutants compared to Gls of mutant alleles of essential and non-essential genes. Red bars indicate the mean. g, Distributions of Pearson correlations between the Gl profiles of Gsp1 interaction partners and Gsp1 mutants if mutation is (right, black) or is not (left, gray) in the interface with that partner. Point size indicates the false discovery rate adjusted one-sided (positive) p-value of the Pearson correlation. Red dots and bars indicate the mean and the upper and lower quartile, respectively. 
Gsp1 switch motif using biophysical studies in vitro (Fig. 1 a, b).

Targeted perturbations to GTPase interaction interfaces. To target each of the 16 known interaction interfaces of Gsp1, we designed 56 S. cerevisiae strains with genomically integrated point mutations in the GSP1 gene (Fig. 1c, Fig. S1, Supplementary File 1 Tables 2, 3). To avoid simultaneously affecting all Gsp1 functions and to create viable mutant strains (as Gsp1 is essential), we excluded mutations in the Gsp1 nucleotide binding site and the switch I and II regions (17). We confirmed by Western blot that the mutant Gsp1 protein levels were close to the endogenous wild-type levels (Fig. S2).

Genetic interactions of Gsp1 mutants. To determine the functional consequences of the Gsp1 interface mutations, we performed a genetic interaction (GI) screen in S. cerevisiae using the epistatic mini-array profile (E-MAP) approach $(18,19)$. We measured growth of each GSP1 point mutant in the context of an array of single gene knockouts, resulting in a quantitative functional profile of up to 1444 GI values for each GSP1 point mutant. Similarity of genetic interaction profiles often indicates shared functions. The 56 GSP1 point mutants fell into two clusters, 23 'strong' mutants with rich GI profiles containing 9-373 significant interactions (Fig. 1d), and 33 'weak' mutants with 0-8 significant interactions (Fig. S3a, Methods and Supplementary File 1 Fig. 1). The strong mutants covered eleven Gsp1 sequence positions and all 16 structurally characterized Gsp1 protein interaction interfaces (Fig. 1e). Remarkably, twelve of the GSP1 interface point mutants had a greater number of significant GIs than an average deletion of a non-essential $S$. cerevisiae gene, and six GSP1 point mutants had more GIs than an average temperature sensitive mutant of an essential gene from a previously published large-scale $S$. cerevisiae GI map (20) (Fig. 1f, Supplementary File 2). The GIs of the designed Gsp1 interface mutations spanned diverse biological processes known to be linked to Gsp1, including mRNA transport, the dynein/dynactin pathway, tRNA modification by the elongator complex, and spindle assembly regulation. Furthermore, unbiased hierarchical clustering of $S$. cerevisiae genes solely by their GI profiles with the 56 GSP1 point mutants also grouped many other genes by their biological complex or pathway membership such as members of the Hog 1 signaling pathway, SWR1 and Rpd3L complexes, and mitochondrial proteins (Fig. 1d, Fig. S3b). Taken together, the GI analysis reveals expansive functional consequences of Gsp1 interface point mutations - similar in magnitude to the effects typically observed for deleting entire genes - that illuminate many of the biological functions of GSP1.

In contrast to their clustering of biological processes, the GI profiles of the Gsp1 point mutants did not group based on their location in the Gsp1 partner interfaces (Fig. 1e). For example, strains with GSP1 mutations at residues Thr34 (T34E, T34Q) and Asp79 (D79S, D79A) have similar GI profiles (Fig. 1d) but these mutations are in different interaction interfaces (Fig. 1e) on opposite sides of the Gsp1 structure (Fig. 1c). This observation was unexpected and contrary to our initial expectation that Gsp1 achieves its functional specificity by interacting with different partners and, accordingly, targeting different protein interfaces should affect distinct functions of Gsp1. To analyze this finding further and quantify the functional similarities between individual GSP1 mutants across most biological processes in S. cerevisiae, we compared the GSP1 mutant GI profiles to profiles from 3358 $S$. cerevisiae genes (20) using Pearson correlations. In this analysis, significant positive correlations signify functional relationships (21) (Supplementary File 3, Supplementary File 1 Table 4, Fig. S3c). Strikingly, GI profiles of GSP1 mutants and of Gsp1 physical interaction partners were on average no more similar to each other in instances where the Gsp1 mutation was located in the partner interface than when the mutation was not (Fig. 1g, Fig. S3d). These results suggest that the rich functional profiles of GSP1 mutants cannot simply be explained by considering only the interface or partner interaction targeted by the point mutation.

Physical interactions of Gsp1 mutants. To investigate further why the GI profiles of Gsp1 mutations did not group based on targeted specific physical interactions of Gsp1, we sought to determine changes to the physical interaction network in response to the mutations (for example, mutations could fail to target the intended interactions). We tagged wild-type Gsp1 and 28 mutants covering all interface residues shown in Fig. 1e with an amino- or carboxyterminal 3xFLAG tag and quantified the abundance of each of the 'prey' partner proteins in complex with Gsp1 by APMS (Fig. 2a, Fig. S4, Supplementary File 4). We refer to the prey partner protein abundance in the pulled-down Gsp1 complexes simply as "abundance" below. As expected, Gsp1 mutations in the cores of interaction interfaces decreased the abundance of the prey partner targeted by each specific mutation (Fig. 2b, large half-circles representing location of the mutation in the interface core are predominantly red, indicating a decreased interaction with the targeted prey partner). However, when considering the changes in abundance of all physical interaction partners observed in the AP-MS experiments (core regulators Rna1 and Srm1, and effectors Yrb1, Kap95, Pse1 and Srp1), we found that several of these interaction partners showed significant changes in interactions with almost all Gsp1 mutants (Fig. 2c). For example, the point mutations at residue Thr34 are in the core of the interface with Yrb1 (Fig. 2d) and accordingly resulted in lower abundance of Yrb1 compared to wild type (Fig. 2b) but also significantly affected the interactions with other partners (Fig. 2c). Over the entire dataset, the most notable change in abundance occurred with the two core GTPase cycle regulators, GAP (Rna1) and GEF (Srm1) (Fig. 2c, Fig. S4d, Supplementary File 1 Table 5), despite most mutations not being in the Rna1 or Srm1 interfaces (Fig. 2d-f). In summary, the AP-MS experiments confirm that the point mutations affected the targeted interactions but also reveal unexpected, extensive changes to the physical interaction network of Gsp1 that cannot simply be explained by the interface location of the mutations.

Molecular mechanism of Gsp1 point mutants. The AP- 
a

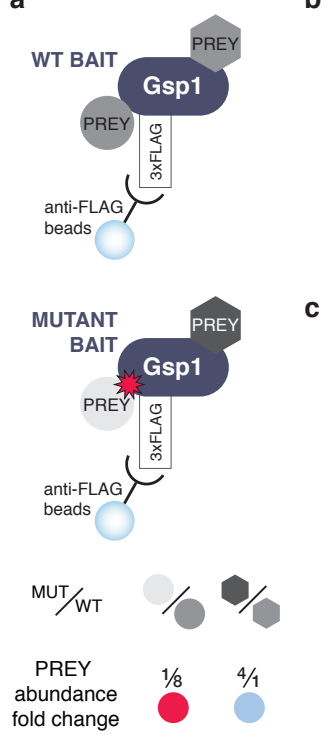

b

c
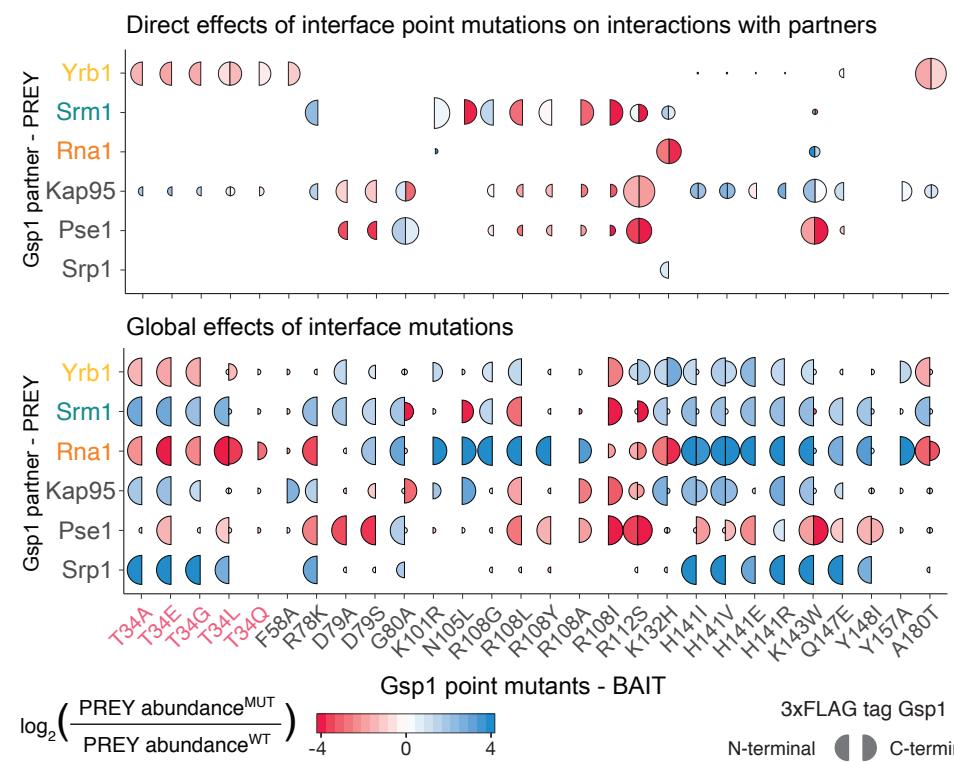

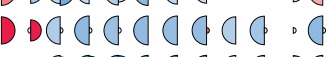

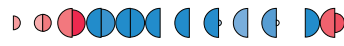

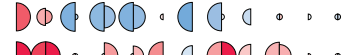
100000

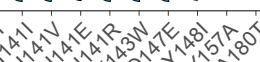

N-terminal C-terminal

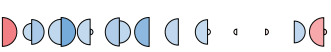

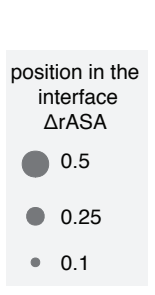

d

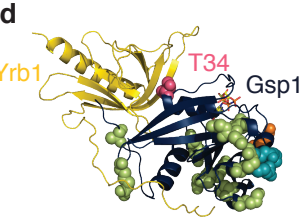

e
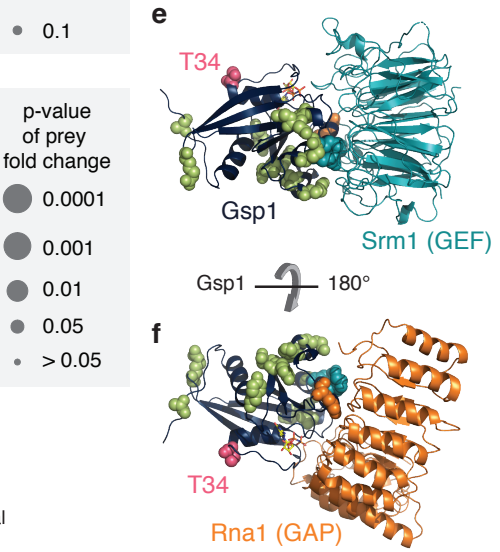

Fig. 2. Gsp1 point mutations in the interfaces with interaction partners globally rewire the physical interaction network of Gsp1, including changes in interactions with the switch regulators GEF (Srm1) and GAP (Rna1). a, Schematic representation of the AP-MS experimental design. b, c, Decreased (red) and increased (blue) abundance of pulled-down physical interaction partner (preys, rows) by mutant Gsp1 (columns) compared to wild type. The log ${ }_{2}$ (PREY abundance ${ }^{\text {MUT }} /$ PREY abundance ${ }^{\text {WT }}$ ) values are capped at +/- 4. Left semi-circle represents an amino-terminal 3xFLAG-tagged Gsp1 mutant, and right semi-circle represents a carboxy-terminal 3xFLAG-tagged Gsp1 mutant. b, Semi-circle size is proportional to change in relative accessible surface area ( $\Delta \mathrm{rASA})$ upon complex formation between Gsp1 and a partner protein. c, Shown are the same data as in b but here semi-circle size is proportional to the significance (false discovery rate adjusted $p$-value) of the fold change of the prey abundance compared to the wild type. $\mathbf{d}$, e, $\mathbf{f}$, Location of Gsp1 mutations with respect to the interfaces with Yrb1 (PDB ID: 1k5d), Rna1 (PDB ID: 1k5d) and Srm1 (PDB ID: 1i2m). Gsp1 residues shown as point mutants in b and c are shown here as spheres, Thr34 (in Yrb1 interface) in pink, residues in the Srm1 interface in teal, residues in the Rna1 interface in orange, and all other mutated residues in green. Gsp1 is in the same orientation in panels $\mathrm{d}$ and $\mathrm{e}$, and it is rotated $180^{\circ}$ around the $\mathrm{x}$-axis in panel $\mathbf{f}$.

MS experiments showed that most Gsp1 interface mutations significantly altered physical interactions with the two principal GTPase regulators, GAP and GEF. To address the question whether the mutations act directly or indirectly (i.e. by altering the competition between physical interaction partners in the cell), we recombinantly expressed and purified wild-type Gsp1 and 22 mutants that expressed and purified well. We then measured the effect of the mutations on GAP-mediated GTP hydrolysis and GEF-mediated nucleotide exchange in vitro (Fig. 3a, b, Fig. S5, Supplementary File 1 Figures 2, 3, and Tables 6, 7). Of the 22 Gsp1 point mutants, 14 (all of which had strong GI profiles except K132H) showed 3- to $>200$-fold effect on either or both of the GAP- or GEF-mediated reactions (Fig. S5e). In particular, mutations that are not in the interface with the GAP both increased (3-fold, R108G mutant) and decreased (10fold, R78K, T34E/Q/A, and D79S mutants) the catalytic efficiency of GAP-mediated GTP hydrolysis, compared to wildtype Gsp1 (Fig. 3a). As expected, mutations in the interface with the GEF (K101, and R108) decreased the catalytic efficiency of GEF-mediated nucleotide exchange $>40$-fold. However, other mutations not in the GEF interface (R78K, Y157A) also decreased the efficiency notably (3- to 10-fold, Fig. 3b). These results show that Gsp1 interface mutations are capable of modulating the GTPase cycle by directly affecting GTP hydrolysis and nucleotide exchange catalyzed by the two core switch regulators, GAP and GEF. Moreover, since seven out of the 14 mutations with larger than 3-fold effects are located outside of the interfaces with either the GAP or the GEF as well as outside the known switch regions, our data suggest considerable, previously unappreciated, allostery in the GTPase switch.

To probe the mechanism of these allosteric effects, we examined the impact of Gsp1 point mutations on the conformational distribution in the active site of GTP-bound Gsp1 using $1 \mathrm{D}^{31} \mathrm{P}$ nuclear magnetic resonance (NMR) spectroscopy. Similar to the human homolog Ran (22), 1D ${ }^{31}$ P NMR spectra of $S$. cerevisiae wild-type Gsp1:GTP showed two distinct peaks for the $\gamma$-phosphate of bound GTP arising from differences in the local chemical environment of the $\gamma$-phosphate in each of two distinct conformations (termed $\gamma 1$ and $\gamma 2$ ) (Fig. S6a). While $87 \%$ of wild-type Gsp1:GTP was in the $\gamma 2$ state conformation, the populations of the $\gamma 2$ state in Gsp1 mutants ranged from close to $0 \%$ for T34E and T34Q, to close to $100 \%$ for H141R, Y157A, and K132H (Fig. 3c). Remarkably, these interface sites are at least $18 \AA$ away from the $\gamma$ phosphate of Gsp1-bound GTP (Fig. 3d, e).

Prior work on human Ran (22) and the related GTPase Ras (23) had implicated the $\gamma 2$ state conformation in effector binding. Here we observe a striking linear relationship when plotting the $\gamma 2$ state population in the different Gsp1 mutants against their effects on relative catalytic efficiency of GAPmediated GTP hydrolysis (Fig. 3f) and intrinsic GTP hydrolysis (Supplementary File 1 Table 8, Fig. S6b, c). These experiments directly quantify the allosteric effects of the mutations and indicate that the $\gamma 2$ state represents the hydrolysiscompetent conformation of Gsp1:GTP. Exceptions to the linear relationship are the $\mathrm{K} 132 \mathrm{H}$ mutation, which is in the core of the GAP interface and is hence expected to directly affect the interaction with the GAP, and the D79S and R78K 
a

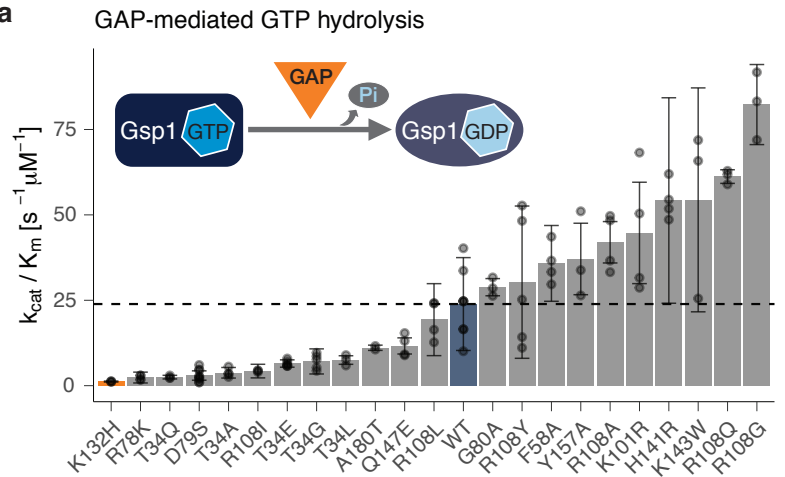

WT Gsp1 $\square$ in GEF interface $\quad$ in GAP interface $\square$ not in either interface

b

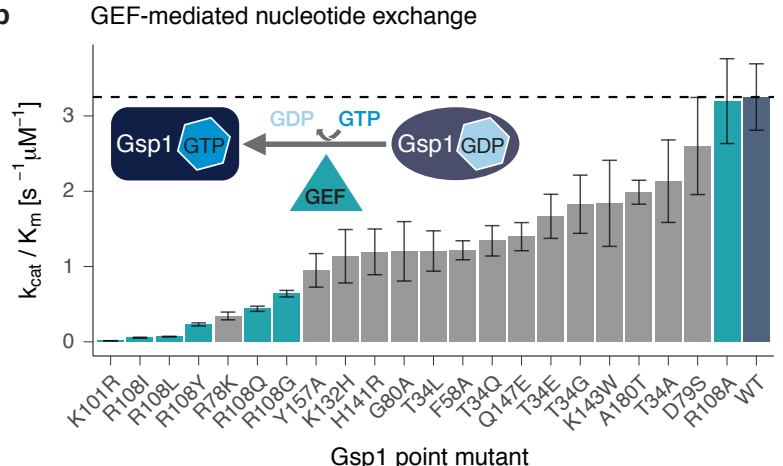

c ${ }^{31}$ P NMR of Gsp1:GTP
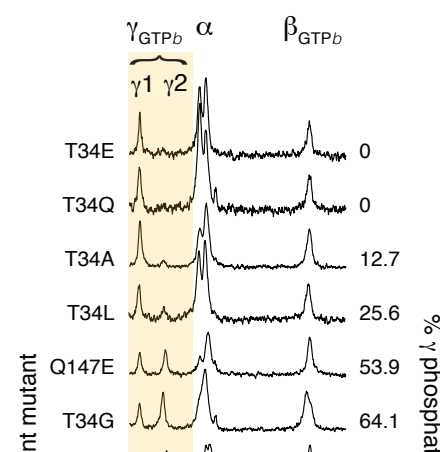

\section{흘}

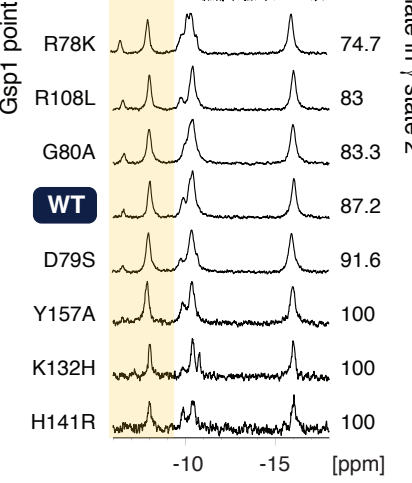

d

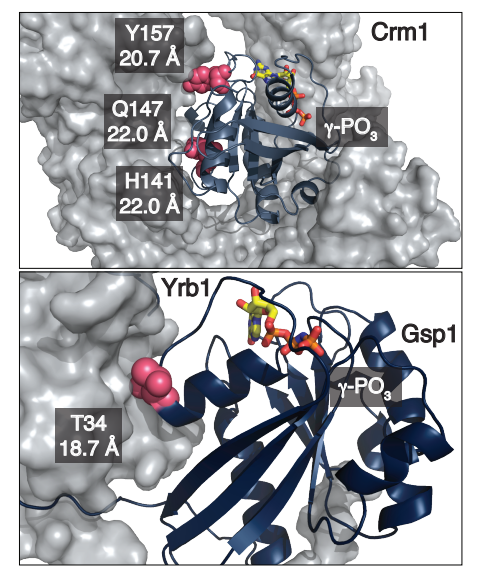

f

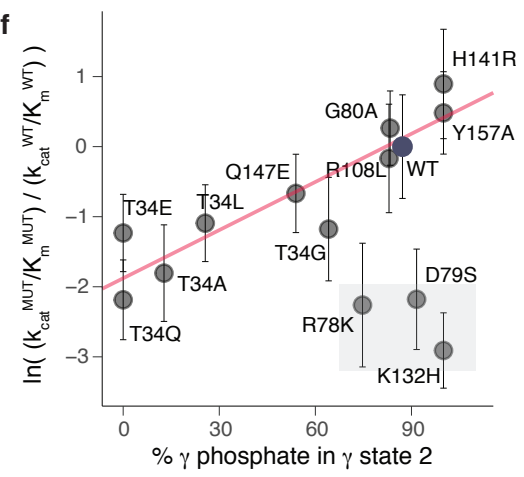

Fig. 3. Point mutations in Gsp1 interfaces allosterically modulate GTPase cycle parameters by tuning active site conformational distributions. a, Catalytic efficiency $\left(\mathrm{k}_{\text {cat }} / \mathrm{K}_{\mathrm{m}}\right)$ of GAP-mediated GTP hydrolysis of Gsp1 mutants. Each individual point represents the ratio of $\mathrm{k}_{\text {cat }}$ and $\mathrm{K}_{\mathrm{m}}$ from an individual GAP-mediated GTP hydrolysis experiment fit to an integrated Michaelis-Menten equation. Error bars represent the combined standard deviations (see Methods) of $k_{c a t}$ and $K_{m}$ from $n 3$ replicates (except for A180T which has two replicates). Dotted line indicates wild-type efficiency. $\mathbf{b}$, Catalytic efficiency ( $\mathrm{k}_{\text {cat }} / \mathrm{K}_{\mathrm{m}}$ ) of GEF-mediated nucleotide exchange of Gsp1 mutants. Error bars represent the value plus/minus the standard error of the Michaelis-Menten fit to data from $n 17$ measurements at different substrate concentrations. $c,{ }^{31} \mathrm{P}$ NMR of GTP bound Gsp1 point mutants. NMR peak heights are normalized to the $\beta$ peak of the bound GTP ( $\beta$ GTPb). The two peaks of the $\gamma$ phosphate of bound GTP are highlighted in yellow. The peak at approximately -7 ppm is defined as $\gamma 1$ and the peak at approximately -8 ppm is defined as $\gamma 2$. The percent of $\gamma$ phosphate in $\gamma 2$ is defined as a ratio of areas under the curve between the $\gamma 2$ and the sum of the $\gamma 1$ and $\gamma 2$ peaks. d, Residues Tyr157, His141, and Gln147 (pink spheres) are in the interface with Crm1 (gray surface, PDB ID: 3m1i). Gsp1 is represented as a navy cartoon and the GTP nucleotide is in yellow stick representation. e, Residue Thr34 (pink spheres) is in the core of the interface with Yrb1 (gray surface, PDB ID: 1k5d). Distances from the $\gamma$ phosphate of GTP to the residue $\alpha$-carbon are indicated below the residue numbers in $d$ and e. $\mathbf{f}$, Percent population in $\gamma 2$ peak plotted against the relative catalytic efficiency of GAP-mediated GTP hydrolysis represented as a natural logarithm of the ratio of the mutant over wild-type $\mathrm{k}_{\mathrm{cat}} / \mathrm{K}_{\mathrm{m}}$. The pink line is a linear fit excluding the three outliers highlighted in the gray box. Error bars represent the standard deviation across at least three replicates of individual GAP-mediated GTP hydrolysis measurements.

mutations, which are on the edge of the GTPase switch II region (from residues 69 to 77 ) and could lead to additional perturbations of the nucleotide binding site geometry. Taken together, the ${ }^{31} \mathrm{P}$ NMR and kinetic data support a molecular mechanism whereby Gsp1 interface mutations allosterically shift the conformational distribution at the active site which in turn alters the GTPase switch cycle. Interestingly, the allosteric mutations we show here to tune the distribution of the catalytically competent state of Gsp1 (T34, H141, Q147, and Y157, Fig. 3d, e) do not overlap with either allosteric inhibitor pocket successfully targeted by small molecule inhibitors in Ras (24-26) (Fig. S6d).

Our in vitro analysis showed that most Gsp1 interface mutations affect GEF-catalyzed nucleotide exchange and GAP-catalyzed GTP hydrolysis differentially (Fig. 3a,b, and Fig. S5e). To determine to what extent these effects explain changes in the physical interaction network of Gsp1 (Fig. 2), we compared in vitro kinetic and our AP-MS data (Fig. 4a). We found that all Gsp1 mutants that affected the efficiency $\left(\mathrm{k}_{\mathrm{cat}} / \mathrm{K}_{\mathrm{m}}\right)$ of GEF-catalyzed nucleotide exchange more than the efficiency of GAP-catalyzed GTP hydrolysis (points above the diagonal in Fig. 4a) showed a larger decrease in the abundance of pulled-down GEF compared to pulled-down GAP (teal points), while the opposite was the case for the mutants below the diagonal (orange points in Fig. 4a). Similar but weaker relationships were observed for other prey proteins (Fig. S7). We conclude that Gsp1 interface mutations allosterically perturb the GTPase cycle, and that the direction of the cycle perturbation is a good predictor of altered physical interactions with the two main cycle regulators, even in the context of many other potentially competing partner proteins.

Encoding of Gsp1 multi-specificity. We next asked whether the allosteric effect of the mutations on the balance of the GTPase cycle, rather than the interface in which a mutation is made, could better explain the functional effects observed in the cellular GI profiles. We clustered the GI profiles of the Gsp1 mutants based on correlation with the GI profiles of 3358 S. cerevisiae genes (20). We then compared clustering of these GI profile correlations (using the 278 genes with significant correlations to Gsp1 mutants, Fig. 4b, Fig. S8a) with the biophysical effects of the mutations on the efficien- 
bioRxiv preprint doi: https://doi.org/10.1101/2020.01.04.893909; this version posted January 6, 2020. The copyright holder for this preprint (which was not certified by peer review) is the author/funder, who has granted bioRxiv a license to display the preprint in perpetuity. It is made available under aCC-BY-NC-ND 4.0 International license.
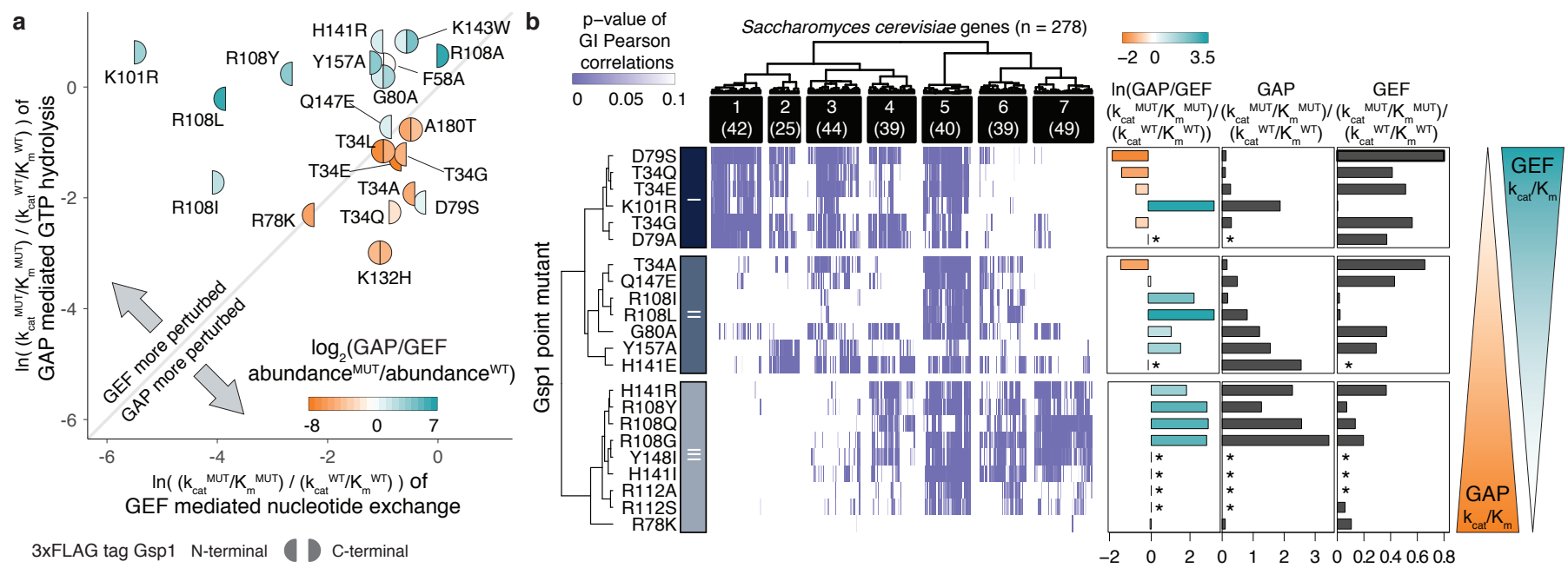

c
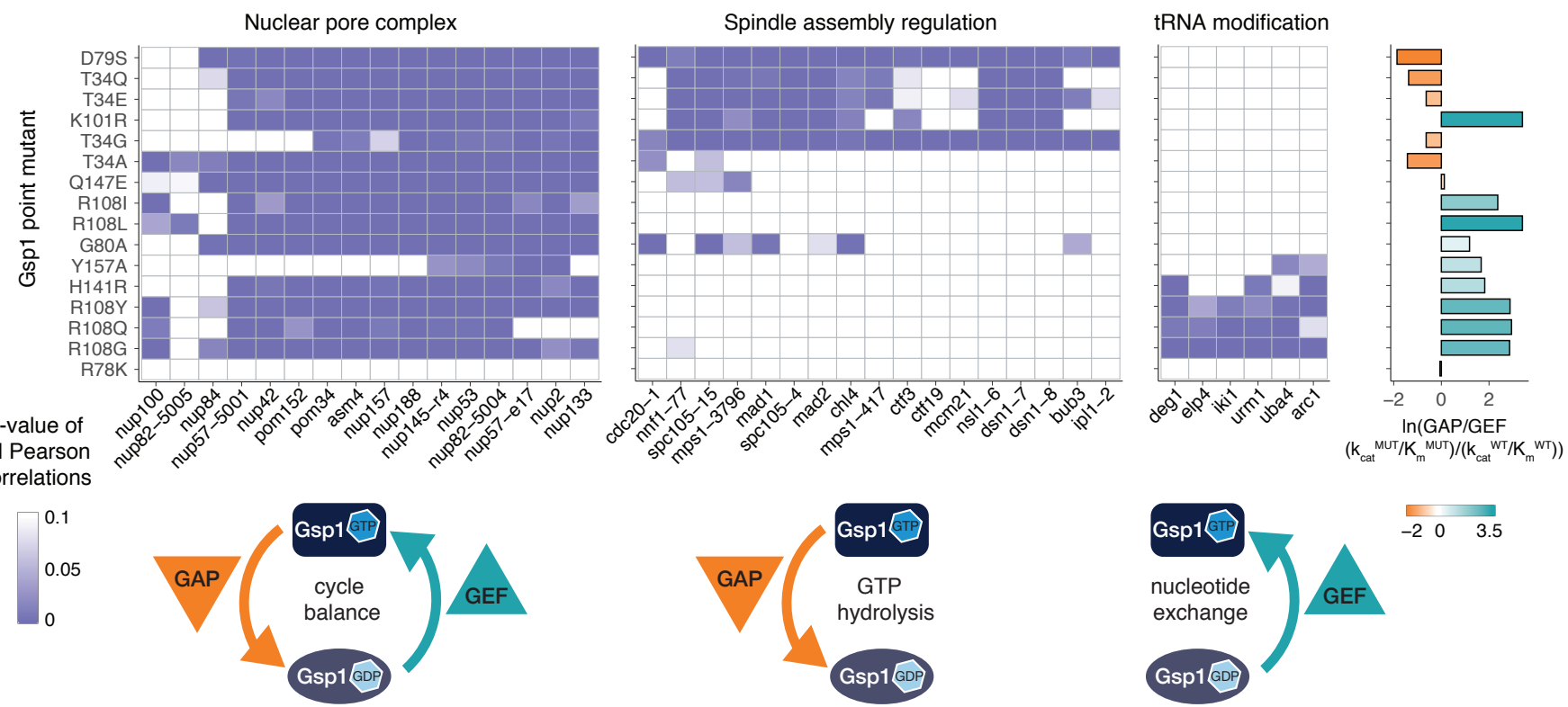

Fig. 4. Cellular effects of interface mutations group by their effect on GTPase cycle kinetics. a, Log 2 -transformed ratio of GAP and GEF abundance fold change for each Gsp1 point mutant compared to wild type from the AP-MS proteomics experiment overlaid onto the relative enzymatic efficiencies of GAP-mediated GTP hydrolysis and GEF-mediated nucleotide exchange. Log $_{2}$-transformed ratio of GAP and GEF abundance fold change is defined as log $2\left((\text { abundance(Rna1 })^{\mathrm{MUT}} / \mathrm{abundance}(\mathrm{Rna} 1)^{\mathrm{WT}}\right) /$ (abundance $(\operatorname{Srm} 1)^{\mathrm{MUT}} /$ abundance $\left.\left.(\mathrm{Srm} 1)^{\mathrm{WT}}\right)\right)$. Relative GAP-mediated hydrolysis and GEF-mediated exchange efficiencies are defined as In $\left(\left(\mathrm{K}_{\mathrm{cat}}{ }^{\mathrm{MUT}} / \mathrm{K}_{\mathrm{m}}{ }^{\mathrm{MUT}}\right) /\left(\mathrm{K}_{\mathrm{cat}}{ }^{\mathrm{WT}} / \mathrm{K}_{\mathrm{m}}{ }^{\mathrm{WT}}\right)\right)$. b, Clustering of 278 S. cerevisiae genes and 22 strong Gsp1 point mutants by the p-value of Pearson correlations of their Gl profiles compared to relative GAP-mediated hydrolysis and GEF-mediated exchange efficiencies as indicated. The p-value is a false discovery rate adjusted one-sided (positive) $p$-value of the Pearson correlations (represented as a white to purple range, where purple is most significant). The number of genes in each of the seven clusters is given in parentheses. A star next to the relative kinetic values indicates that no kinetics data were collected for that mutant. c, $S$. cerevisiae nuclear pore genes show significant Pearson correlations (false discovery rate adjusted one-sided (positive) p-value $<0.05$ ) with mutants regardless of which side of the GTPase cycle is more perturbed, while genes involved in spindle assembly regulation and tRNA modification correlate significantly with mutants that are more perturbed in GAP-mediated hydrolysis and GEF-mediated nucleotide exchange, respectively. The order of Gsp1 mutants with available kinetics data corresponds to the order in panel b. S. cerevisiae genes are clustered by p-value. The GTPase cycle schemes below the heatmaps represent each of the three paradigms of Gsp1 function.

cies of GAP-catalyzed GTP hydrolysis and GEF-catalyzed nucleotide exchange. Remarkably, the Gsp1 mutant GI profile clustering mirrored an approximate ordering by the in vitro mutant effects on the GTPase cycle: relative GEF efficiency systematically decreased with increasing row number and relative GAP efficiency systematically increased (Fig. 4b). A clear outlier of this ordering is the K101R mutant, which primarily affects GEF-mediated nucleotide exchange in vitro but, by GI profiles, groups with mutations affecting the efficiency of GTP hydrolysis. A lysine at the corresponding position (Lys99) is annotated to be acetylated in mam- malian systems (27), and this acetylation was shown to reduce the efficiency of nucleotide release from Ran:GDP:GEF complexes (28). We hypothesize that while our K101R mutation affected the interaction with the GEF, it also likely broke a critical mechanism by which the cell reduces GEF activity, phenocopying the mutants with reduced GTP hydrolysis activity. This observation suggests the possibility that the allosteric sites discovered here might be used to control cellular functions via posttranslational modifications.

Finally, we asked whether our biophysical measurements of how the different Gsp1 mutants perturb the GTPase cy- 
cle regulation could provide insight into Gsp1's functional multi-specificity, i.e. its ability to distinctly regulate multiple biological processes. Clustering the S. cerevisiae genes and Gsp1 mutants based on correlations of their respective GI profiles revealed that genes fall into one of three categories (Fig. 4b, Fig. S8a): (i) genes that correlate strongly with all or most of the Gsp1 point mutants (most strikingly genes in cluster 5, but also clusters 3,4 , and 6), irrespective of the direction of GTPase cycle perturbation; (ii) genes in cluster 1 , but also cluster 2 , that correlate primarily with mutants more perturbed in the GTP hydrolysis side of the cycle (orange bars) and (iii) genes in cluster 7 that correlate primarily with mutants more perturbed in the nucleotide exchange side of the cycle (teal bars). Most importantly, this clustering distinguishes between biological processes, since genes with shared biological function (Supplementary File 5) all predominantly fall into one of the three categories defined above (Fig. 4c, Fig. S8b-e and 9). Overall, our analysis suggests that distinct processes regulated by Gsp1, such as nuclear transport, spindle assembly, and tRNA modification (Fig. 4c) as well as 5 mRNA capping, transcription regulation, and cell polarity (Fig. S9b-d) are differentially sensitive to perturbations of each side of the cycle.

Discussion. Only five years after the discovery of the small GTPase Ran, Rush et al. (29) proposed that Ran must act by two different mechanisms: one in which the cycling of the GTPase is most important ('Rab paradigm'), and the other in which the amount of "active" Ran:GTP is most important ('Ras paradigm'). Our findings lead to a model where Ran/Gsp1 acts by three different paradigms that are defined by the sensitivity of different biological processes to perturbations of different characteristics of the Gsp1 GTPase cycle, i.e. the ability to (i) cycle, (ii) turn off by hydrolyzing to Gsp1:GDP, and (iii) activate by producing Gsp1:GTP. Other effects such as direct perturbations of interactions and binding partner competition undoubtedly also play a role in modulating the detailed effects of our Gsp1 mutations. Nevertheless, our model explains to a remarkable degree how a single molecular switch motif can differentially control subsets of biological processes by using one of the three functional modes.

The discovery of several allosteric sites (positions 34, 141, 147, and 157) in the model molecular switch Gsp1 both explains the widespread functional consequences we observe for single amino acid point mutations at interaction surfaces of Gsp1 and has important implications for revising our understanding of GTPase switch regulation. We show that mutations in distal interaction interfaces allosterically modulate the switch cycle. This finding demonstrates thermodynamic coupling between interaction interfaces and the classical switch region in the active site and thereby suggests that partners binding to distal sites also regulate the switch by affecting conformational equilibria at the active site. This hypothesis is supported by evidence that the Yrb1 homolog RanBP1 modulates GAP activity $(22,30,31)$. Our data provide a mechanistic explanation, where mutations at allosteric sites, including Thr34 in the Yrb1 binding interface, tune the population of Gsp1 in a hydrolytically-primed conformation. Since the overall switch mechanism is conserved across the small GTPase fold, we propose that thermodynamic coupling between distal interfaces and functional conformational changes may be a more general mechanism to regulate other GTPase switches, and may aid in the development of allosteric inhibitors.

Our observation of widespread functional effects induced by relatively small mutational perturbations is reminiscent of the ultrasensitivity achievable in biological motifs with opposing regulators (6). While switch-like ultrasensitivity is typically described for systems controlled by covalent modifications (such as phosphorylation), our results, as well as the observations that cellular levels of small GTPase regulators require tight control $(32,33)$, corroborate a model of ultrasensitivity for GTPase conformational switches (34). While we investigated the changes to the GTPase cycle caused by mutations, similar effects on regulation could be exerted by partner binding or posttranslational modification.

Finally, deriving a model that explains the cellular multispecificity of GTPases by differential sensitivity of biological processes to distinct parameters of the switch cycle was enabled by a quantitative analysis that integrated functional genomics, proteomics, and biophysics. Given the prevalence of biological two-state switch motifs controlled by opposing regulators (kinase/phosphatase, acetylase/deacetylase) (35), we envision this approach to be fruitful for other studies of cellular regulation and to be extended to mammalian systems using CRISPR-based approaches to yield mechanistic insights into the drastic consequences of disease mutations targeting central molecular switches.

\section{Methods}

Point mutations in genomic Gsp1 sequence. We identified all residues in Gsp1 that comprised the interfaces with Gsp1 binding partners for which co-complex crystal structures with Gsp1 were available (Supplementary File 1 Table 1, Fig. S1a). Residues comprising interface core, support or rim were defined based on relative change in solvent accessible surface area ( $\triangle$ rASA), as previously defined (5), compared to the empirical maximum solvent accessible surface area for each of the 20 amino acids (36). Accessible surface area was calculated using the bio3d R package (37). All the custom code for interface analysis from co-complex crystal structures is provided in the associated code repository in the structure analysis folder. We avoided Gsp1 residues that are within $5 \AA$ of the nucleotide (GDP or GTP) in any of the structures or that are within the canonical small GTPase switch regions (P-loop, switch loop I, and switch loop II). We then mutated residues that are located in interface cores (defined as residues that bury more than $25 \%$ of their surface upon complex formation, as previously defined (5), Supplementary File 1 Table 2, Fig. S1b) into amino acid residues with a range of properties (differing in size, charge and polarity) and attempted to make stable and viable $S$. cerevisiae strains carrying a genomic Gsp1 point mutation coupled to nourseothricin (clonNAT / nourseothricin, Werner BioAgents 
GmbH, CAS 96736-11-7) resistance. The list of attempted mutants is provided in Supplementary File 1 Table 3. The genomic construct was designed to minimally disrupt the non-coding sequences known at the time, including the 5 UTR and 3 UTR, as well as the putative regulatory elements in the downstream gene Sec72 (Supplementary File 1 Fig. 4). The GSP1 genomic region was cloned into a pCR2.1TOPO vector (Invitrogen) and point mutations in the GSPI coding sequence were introduced using the QuikChange ${ }^{\mathrm{TM}}$ Site-Directed Mutagenesis (Stratagene, La Jolla) protocol. $S$. cerevisiae strains containing mutant GSP1 genes were regularly confirmed by sequencing the Gsp1 genomic region.

\section{S. cerevisiae genetics and genetic interaction mapping.}

$S$. cerevisiae transformation. To generate MAT: $\alpha$ strains with Gsp1 point mutations the entire cassette was amplified by PCR using $S$. cerevisiae transformation forward and reverse primers, and $S$. cerevisiae was transformed into the starting SGA MAT: $\alpha$ his3D1; leu2D0; ura3D0; LYS2p; can1::STE2pr-SpHIS5 (SpHIS5 is the $S$. pombe HIS5 gene); lyp1D::STE3pr-LEU2 strain from (38) as described below. Primers for amplifying the GSP1 genomic region: S. cerevisiae Transformation FWD: GTATGATCAACTTTTCCTCACCTTTTAAGTTTGTTTCG $S$. cerevisiae Transformation REV: GATTGGAGAAACCAACCCAAATTTTACACCACAA

DNA competent $S$. cerevisiae cells were made using a LiAc protocol. The final transformation mixture contained $10 \mathrm{mM}$ LiAc (Lithium acetate dihydrate, 98\%, extra pure, ACROS Organics TM, CAS 6108-17-4), $50 \mu \mathrm{g}$ ssDNA (UltraPure $^{\mathrm{TM}}$ Salmon Sperm DNA Solution, Invitrogen, 15632011), 30\% sterile-filtered PEG 8000 (Poly(ethylene glycol), BioUltra, 8,000, Sigma-Aldrich, 89510-250G-F). A $S$. cerevisiae pellet of approximately $25 \mu \mathrm{l}$ was mixed with $15 \mu \mathrm{l}$ of linear DNA PCR product and $240 \mu \mathrm{l}$ of the transformation mixture, and heat shocked at $42^{\circ} \mathrm{C}$ for 40 minutes. Transformed cells were grown on YPD (20 g Bacto ${ }^{\text {TM }}$ Peptone (CAT \# 211820, BD Diagnostic Systems), $10 \mathrm{~g}$ Bacto $^{\mathrm{TM}}$ Yeast Extract (CAT \# 212720 BD), and $20 \mathrm{~g}$ Dextrose (CAT \# D16-3, Fisher Chemicals) per 1-liter medium) + clonNAT plates and incubated at $30^{\circ} \mathrm{C}$ for 3 to 6 days. Many colonies that appeared after 24-48 hours carried the clonNAT cassette but not the GSP1 point mutation, or the 3xFLAG tag. Cells were therefore sparsely plated and plates were incubated for a longer period of time after which colonies of different sizes were picked and the mutant strains were confirmed by sequencing.

Epistatic mini-array profiling (E-MAP) of Gsp1 point mutants. Genetic interactions of all viable GSP1 point mutant (PM-GSP1-clonNAT) strains were identified by epistatic miniarray profile (E-MAP) screens $(19,38)$ using a previously constructed array library of 1,536 KAN-marked (kanamycin) mutant strains assembled from the S. cerevisiae deletion collection (39) and the DAmP (decreased abundance by mRNA perturbation ) strain collection (40), covering genes involved in a wide variety of cellular processes $(18,41)$. The E-MAP screen was conducted as previously described in Collins et al. (38), using the HT Colony Grid
Analyzer Java program (21) and E-MAP toolbox for MAT$\mathrm{LAB}$ to extract colony sizes of double mutant strains and a statistical scoring scheme to compute genetic interaction scores. Genetic interaction scores represent the average of 3-5 independent replicate screens. Reproducibility was assessed as previously described (21) by comparing individual scores to the average score for each mutant:gene pair, with the two values showing strong correlation across the dataset (Pearson correlation coefficient $=0.83$, Supplementary File 1 Figure 5).

Hierarchical clustering of E-MAP genetic interaction data. All E-MAP library DAmP strains as well as library strains showing poor reproducibility were discarded, leaving 1444 out of the original 1536 library genes. Averaged S-scores of genetic interactions between wild type and point mutant Gsp1 and the 1444 S. cerevisiae genes are provided in Supplementary File 2. Hierarchical clustering on the genetic interaction profiles was performed using the average linkage method and the pairwise Pearson correlation coefficient as a distance metric. To identify clusters of functionally related library genes, the hierarchical clustering tree was cut to produce 1200 clusters, resulting in 43 clusters with 3 or more members. Biological function descriptions for genes in these clusters were extracted from the Saccharomyces Genome Database (SGD) (42). Clusters of genes representing common functions (complexes, pathways or biological functions) were selected by manual inspection and represented in the main text Fig. 1d and Fig. S3b. All of the custom code for E-MAP analysis is provided in the EMAP analysis GitHub repository. Clustered heatmaps were produced using the ComplexHeatmap package (43).

Scaling of published genetic interaction data to the EMAP format. To enable comparison of GSP1 point mutant genetic interaction profiles to profiles of other $S$. cerevisiae genes, published Synthetic Gene Array (SGA) genetic interaction data (20) from CellMap.org (44) were scaled to the E-MAP format using a published non-linear scaling method (45). Briefly, 75,314 genetic interaction pairs present in both the SGA and a previously described E-MAP dataset used to study chromatin biology (46) were ordered by genetic interaction score and partitioned into 500 equally sized bins separately for each dataset. Bin size (150 pairs per bin) was chosen to provide enough bins for fitting the scaling spline (described below) while still maintaining a large number of pairs per bin such that the mean could be used as a high confidence estimate of the score values in each bin. Scaling factors were computed that scaled the mean of each SGA bin to match the mean of the corresponding E-MAP bin. A non-linear univariate spline was fit through the scaling factors, providing a scaling function that was subsequently applied to each SGA score. The distribution of scores of shared interactions between the scaled SGA and the E-MAP chromatin library was similar to that between replicates in the E-MAP chromatin library, matching what was seen in the previously published scaling of SGA data to E-MAP format (45) (Supplementary File 1 Fig. 6). The SGA genetic interaction scores are taken from CellMap.org (44). The scaling code is provided in SGA 
scaling GitHub repository.

Significance of genetic interactions. The S-score metric used in scoring genetic interactions measured by the E-MAP method has been previously characterized in terms of confidence that any given averaged S-score represents a significant interaction (21). We fit a spline to data points from Fig. 4c from Collins et al (21), allowing us to provide an approximate confidence estimate for each of our measured GSP1 and scaled S. cerevisiae SGA genetic interaction scores. The SGA dataset (20) is accompanied by p-values as well as its own recommendations for a threshold at which individual interactions are considered significant. We plotted the SGA score scaled to E-MAP format vs. the associated p-value (negative log-transformed, Supplementary File 1 Fig. 1a) and found the distribution to have a similar shape to the confidence function for S-scores (Supplementary File 1 Fig. 1b). For example, a $95 \%$ confidence threshold is associated with E-MAP S-scores less than -4 or greater than 5, while the median p-value of scaled SGA scores is less than 0.05 for scores less than -5 or greater than 3 . We ultimately elected to use a significance cutoff of absolute $\mathrm{S}$-score greater than 3 . This threshold corresponds to an estimated confidence value of 0.83 for S-scores less than -3 and 0.65 for S-scores greater than 3 . We compared these values to the intermediate significance threshold recommended for the SGA data from Ref. (20), which was p-value $<0.05$ and absolute SGA score $>$ 0.08. After scaling to E-MAP format, this threshold corresponds to scaled S-scores less than -2.97 or greater than 2.25 , below our chosen threshold of -3 and 3 .

GI profile correlation measurements Of the 1444 library genes in the GSP1 point mutant GI profile map, 1129 were present in the SGA dataset from Ref. (20). Pairwise Pearson correlation coefficients were computed between all GSP1 point mutants and SGA gene profiles, and all profiles trimmed to include only genetic interaction measurements with the 1129 shared library genes. Due to the relative sparsity of genetic interaction profiles, pairwise comparisons are dominated by high numbers of non-significant interactions. Accordingly, we did not consider correlations with GSP1 point mutants or SGA gene profiles that did not have significant genetic interactions (absolute scaled S-score greater than 3, see above) with at least 10 of the 1129 library genes. This requirement removed all weak Gsp1 point mutants and one strong mutant (R108A) from the correlation analysis (as they had at most nine genetic interactions with absolute score greater than 3 ), leaving 22 strong mutants and 3383 S. cerevisiae SGA genes to be included in the correlation analysis. All Pearson correlations and their p-values between Gsp1 mutants and S. cerevisiae genes, including all correlations that did not pass our significance filtering procedures, are provided in Supplementary File 3. The subset of Pearson correlations between Gsp1 point mutants and Gsp1 partners with available co-complex X-ray crystal structures, used to make the point plots in Figs. $1 \mathbf{g}$ and $\mathbf{7 c}$, $\mathbf{d}$, are also available in Supplementary File 1 Table 4. Statistical significance of correlations was computed using both two-sided and one-sided (positive) t-tests adjusted for multiple hypoth- esis testing using both the Bonferroni method and the FDR method, which controls the false discovery rate (47). All pvalues reported in the text and figures are one-sided (positive) and corrected by the FDR method, unless otherwise stated. Custom code for genetic interaction profile correlation calculations and filtering is provided in the accompanying E-MAP correlation GitHub repository.

Significance testing was used to filter out $S$. cerevisiae gene SGA profiles that did not show a significant correlation (one-sided positive, Bonferroni-adjusted) with at least two GSP1 point mutants profile. In total, 278 S. cerevisiae genes from the SGA had a significant profile correlation (one-sided positive, Bonferroni-adjusted) with at least two GSP1 point mutants and were therefore included in the correlation analysis shown in Fig. 4b and Fig. S8a. After this filtering step, the one-sided p-values were used to populate a matrix of 22 mutants vs. 278 genes, and hierarchical clustering was performed using Ward's method. Pearson correlation between correlation vectors was used as a distance metric for the mutant (row) clustering, while Euclidean distance was selected for the gene (column) clustering, due to the column vectors being relatively short (22 mutants per column vs. 278 genes per row) and thus sensitive to outliers when clustered using Pearson correlations as the distance metric.

For the gene set analysis we decreased the stringency of inclusion of $S$. cerevisiae SGA genes to include all genes with a significant profile correlation (one-sided positive, Bonferroni-adjusted) with one or more Gsp1 mutants, which added another 201 genes, resulting in 479 genes. We made the gene sets larger to increase our confidence in connecting the patterns of correlations between $S$. cerevisiae genes and Gsp1 mutants, and GTPase cycle parameters represented in Fig. 4c and Fig. S9. Manually curated gene sets of S. cerevisiae genes with significant correlations with Gsp1 mutants are provided in Supplementary File 5.

Protein expression levels by Western Blot. S. cerevisiae strains were grown at $30^{\circ} \mathrm{C}$ in YPD medium $\left(20 \mathrm{~g} \mathrm{Bacto}^{\mathrm{TM}}\right.$ Peptone (CAT \# 211820, BD Diagnostic Systems), $10 \mathrm{~g}$ Bacto $^{\text {TM }}$ Yeast Extract (CAT \# 212720 BD), and 20 g Dextrose (CAT \# D16-3, Fisher Chemicals) per $1 \mathrm{~L}$ medium) for 1.5 - 2 hours until $\mathrm{OD}_{600}$ reached 0.3. Cell culture aliquots of $1 \mathrm{ml}$ were centrifuged for 3 minutes at $21,000 \mathrm{x} \mathrm{g}$ and resuspended in $30 \mu \mathrm{l}$ of phosphate buffered saline $(137 \mathrm{mM}$ $\mathrm{NaCl}, 2.7 \mathrm{mM} \mathrm{KCl}, 10 \mathrm{mM} \mathrm{Na} \mathrm{HPO}_{4}, 1.8 \mathrm{mM} \mathrm{KH}_{2} \mathrm{PO}_{4}$, $\mathrm{pH}=7.4$ ) and $10 \mu \mathrm{l}$ of SDS-PAGE Sample Buffer (CAT \# 161-0747, BioRad), to a final SDS concentration of $1 \%$, and $2 \mathrm{mM}$ beta-mercaptoethanol. Lysates were run $(3 \mu \mathrm{l}$ for most, and $6 \mu \mathrm{l}$ for slow growing mutants with lower OD600) on Stain-Free gels (4-20\%, CAT \# 4568096, BioRad, Tris/Glycine SDS Buffer (CAT \# 161-0732, BioRad)). After electrophoresis, the gel was scanned for total protein quantification and the proteins were subsequently transferred to an Immobilon-FL PVDF membrane (CAT \# IPF00010, EMD Millipore). The membrane was probed with Rabbit anti-RAN (CAT \# PA 1-5783, ThermoFisher Scientific) primary, and Goat anti-Rabbit-IgG(H+L)-HRP (CAT \# 31460, Thermo Fisher) secondary antibodies. The membrane was 
developed using Super Signal West Femto substrate (CAT \# 34096, Thermo Fisher), and scanned and analyzed with Image Lab software on a ChemiDoc MP (BioRad). Each blot had at least one wild-type (WT-GSP1-clonNAT) and at least one MAT: $\alpha$ strain control. The total protein levels (TPMUT) for each Gsp1 point mutant lane were then normalized to the wild-type (WT-GSP1-clonNAT) lane of the corresponding blot $\left(\mathrm{TP}^{\mathrm{WT}}\right)$, providing an adjustment value to account for differences in loading between lanes

$$
a^{M U T}=\frac{T P^{M U T}}{T P^{W T}} .
$$

To compute the relative expression of a Gsp1 point mutant, the density ( $\mathrm{D}^{\mathrm{MUT}}$ ) of the Western blot bands corresponding to the Gsp1 point mutant was divided by the total protein adjustment and finally normalized against the same value for the wild type Gsp1, i.e.

$$
\text { rel.expression }{ }^{M U T}=\frac{\frac{D^{M U T}}{a^{M U T}}}{\frac{D^{W T}}{a^{W T}}} .
$$

Note that for blots with a single WT lane, $\mathrm{a}^{\mathrm{WT}}=1$. For blots with more than one WT lane included, $\mathrm{a}^{\mathrm{WT}}$ was computed for each WT lane by normalizing to the average TP across all WT lanes, and the average adjusted WT density $\left(\mathrm{D}^{\mathrm{WT}} / \mathrm{a}^{\mathrm{WT}}\right.$ ) across all WT lanes was used for computing the relative expression of point mutants. Example Western blots are provided in Supplementary File 1 Fig. 7, and the final protein expression level data for all the mutants are shown in Fig. S2. Identifying Gsp1 complexes in $S$. cerevisiae with affinity purification mass spectrometry (AP-MS)

S. cerevisiae cell lysate preparation. S. cerevisiae strains for AP-MS were grown in YAPD medium (120 mg adenine hemisulfate salt (CAT \# A9126, SIGMA), 10 g Bacto yeast extract (CAT \# BD 212720), $20 \mathrm{~g}$ Bacto peptone (CAT \# BD 211820), 20 g dextrose (D-glucose D16-3 Fisher Chemicals) per $1 \mathrm{~L}$ of medium). Each strain was grown at $30^{\circ} \mathrm{C}$ for 12 to $24 \mathrm{~h}$ to $\mathrm{OD}_{600}$ of 1-1.5. The cells were harvested by centrifugation at $3000 \mathrm{RCF}$ for 3 minutes and the pellet was washed in $50 \mathrm{ml}$ of ice-cold $\mathrm{ddH}_{2} \mathrm{O}$, followed by a wash in $50 \mathrm{ml}$ of $2 \mathrm{x}$ lysis buffer (200 mM HEPES pH 7.5, $200 \mathrm{mM} \mathrm{KCl,} 2$ $\mathrm{mM} \mathrm{MgCl} 2,30 \mu \mathrm{M}$ GTP (Guanosine 5-triphosphate sodium salt hydrate, CAT \# G8877, Sigma-Aldrich), 1 mM Dithiothreitol (Promega V3151), 0.1\% IGEPAL CA-630 (CAT \# I8896, Sigma-Aldrich), and 10\% glycerol). Each pellet of approximately $500 \mu \mathrm{l}$ was then resuspended in $500 \mu \mathrm{l}$ of $2 \mathrm{X}$ lysis buffer supplemented with protease inhibitors without EDTA (cOmplete, Mini, EDTA-free Protease Inhibitor Cocktail, CAT \# 11836170001, Roche) and dripped through a syringe into liquid nitrogen. The frozen $S$. cerevisiae cell pellets were lysed in liquid nitrogen with a SPEX ${ }^{\mathrm{TM}}$ SamplePrep 6870 Freezer/MillTM.

FLAG immunoprecipitation. FLAG immunoprecipitations were performed as previously described $(48,49)$. Details are as follows. For FLAG immunoprecipitations, frozen samples were initially kept at room temperature for 5 minutes and then placed on ice or at $4^{\circ} \mathrm{C}$ in all subsequent steps, unless indicated otherwise. Following the addition of 1.5 - $3.0 \mathrm{ml}$ Suspension Buffer (0.1 M HEPES pH 7.5, 0.1 M $\mathrm{KCl}, 1 \mathrm{mM} \mathrm{MgCl} 2,15 \mu \mathrm{M}$ GTP, and $0.5 \mathrm{mM}$ Dithiothreitol) supplemented with cOmplete mini EDTA-free protease and PhosSTOP phosphatase inhibitor cocktails (Roche), samples were incubated on a rotator for at least 10 minutes and then adjusted to $6 \mathrm{ml}$ total volume with additional Suspension Buffer supplemented with inhibitors before centrifugation at 18,000 rpm for 10 minutes. Anti-FLAG M2 Affinity Gel beads (50 $\mu$ l slurry; Sigma-Aldrich) were washed twice with $1 \mathrm{ml}$ Suspension Buffer. After reserving $50 \mu \mathrm{l}$, the remaining supernatant and anti-FLAG M2 Affinity Gel beads were combined and incubated for 2 hours on a tube rotator. Beads were then collected by centrifugation at $300 \mathrm{rpm}$ for 5 minutes and washed three times. For each wash step, beads were alternately suspended in $1 \mathrm{ml}$ Suspension Buffer and collected by centrifugation at 2,000 rpm for 5 minutes. After removing residual wash buffer, proteins were eluted in $42 \mu \mathrm{l} 0.1 \mathrm{mg} / \mathrm{ml}$ 3xFLAG peptide, $0.05 \%$ RapiGest SF Surfactant (Waters Corporation) in Suspension Buffer by gently agitating beads on a vortex mixer at room temperature for 30 minutes. Immunoprecipitated proteins ( $4 \mu \mathrm{l}$ ) were resolved on 4-20\% Criterion Tris-HCl Precast gels (BioRad) and visualized by silver stain (Pierce Silver Stain Kit; Thermo Scientific) (Supplementary File 1 Fig. 8) before submitting $10 \mu \mathrm{l}$ of each sample for mass spectrometry. At least three independent biological replicates were performed for each FLAG-tagged protein and the untagged negative control.

Liquid chromatography with tandem mass spectrometry (LC-MS/MS) analysis. To prepare samples for LC-MS/MS analysis, immunoprecipitated protein $(10 \mu \mathrm{l})$ was denatured

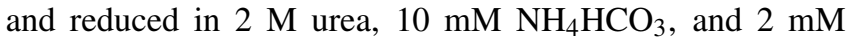
Dithiothreitol for 30 minutes at $60^{\circ} \mathrm{C}$ with constant shaking, alkylated in the dark with $2 \mathrm{mM}$ iodoacetamide for 45 minutes at room temperature and digested overnight at $37^{\circ} \mathrm{C}$ with $80 \mathrm{ng}$ trypsin (Promega). Following digestion, peptides were acidified with formic acid and desalted using C18 ZipTips (Millipore) according to the manufacturer's specifications. Samples were re-suspended in $4 \%$ formic acid, $2 \%$ acetonitrile solution, and separated by a 75-minute reversedphase gradient over a nanoflow C18 column (Dr. Maisch). Peptides were directly injected into a Q-Exactive Plus mass spectrometer (Thermo), with all MS1 and MS2 spectra collected in the orbitrap. Raw MS data were searched against the $S$. cerevisiae proteome (SGD sequences downloaded January 13, 2015) using the default settings in MaxQuant (version 1.5.7.4), with a match-between-runs enabled $(50,51)$. Peptides and proteins were filtered to $1 \%$ false discovery rate in MaxQuant, and identified proteins were then subjected to protein-protein interaction scoring using SAINTexpress (52). Label free quantification and statistical analysis were performed using MSstats (53). Fold change in abundance of preys for 3xFLAG-tagged Gsp1 point mutants was always calculated compared to the wild type Gsp1 with the corresponding tag. All AP-MS data are available from the PRIDE repository under the PXD016338 identifier. Custom code for analysis of AP-MS data is available from the accompanying 
AP-MS GitHub repository. Fold change values between prey abundance between the mutant and wild type Gsp1 and the corresponding FDR adjusted p-values are provided in Supplementary File 4. The intersection of all prey proteins identified at least once with both the amino- or carboxy-terminal $3 x F L A G$ tag, and their interquartile ranges (IQR) of $\log _{2}$ (fold change) values across all the Gsp1 mutants, are provided in Supplementary File 1 Table 5. Quality of data and reproducibility between replicates was assessed based on correlations of peptide counts between replicates (Supplementary File 1 Figs. 9, 10).

\section{Biochemical and biophysical assays.}

Protein purifications. All proteins were expressed from a pET-28 a (+) vector with a N-terminal 6xHis tag in E. coli strain BL21 (DE3) in the presence of $50 \mathrm{mg} / \mathrm{L}$ Kanamycin. GEF (Srm1 from S. cerevisiae, (Uniprot P21827)) was purified as $\Delta 1-27 \mathrm{Srm} 1$ and GAP (Rna1 from $S$. pombe, Uniprot $\mathrm{P} 41391)$ as a full-length protein. $\mathrm{Sc} \Delta 1-27 \mathrm{Srm} 1$ and SpRna1 were expressed in 2xYT medium $(10 \mathrm{~g} \mathrm{NaCl}, 10 \mathrm{~g}$ yeast extract (BD BactoTM Yeast Extract \#212720), 16 g tryptone (Fisher, BP1421) per $1 \mathrm{~L}$ of medium) overnight at $25^{\circ} \mathrm{C}$ upon addition of $300 \mu \mathrm{mol} / \mathrm{L}$ Isopropyl- $\beta$-D-thiogalactoside (IPTG). Gsp1 variants were expressed by autoinduction for 60 hours at $20^{\circ} \mathrm{C}(54)$. The autoinduction medium consisted of $\mathrm{ZY}$ medium (10 g/L tryptone, $5 \mathrm{~g} / \mathrm{L}$ yeast extract) supplemented with the following stock mixtures: 20xNPS (1M $\mathrm{Na}_{2} \mathrm{HPO}_{4}, 1 \mathrm{M} \mathrm{KH}_{2} \mathrm{PO}_{4}$, and $\left.0.5 \mathrm{M}\left(\mathrm{NH}_{4}\right)_{2} \mathrm{SO}_{4}\right), 50 \mathrm{x} 5052$ (25\% glycerol, $2.5 \%$ glucose, and $10 \% \alpha$-lactose monohydrate), 1000x trace metal mixture $\left(50 \mathrm{mM} \mathrm{FeCl}{ }_{3}, 20 \mathrm{mM}\right.$ $\mathrm{CaCl}_{2}, 10 \mathrm{mM}$ each of $\mathrm{MnCl}_{2}$ and $\mathrm{ZnSO}_{4}$, and $2 \mathrm{mM}$ each of $\mathrm{CoCl}_{2}, \mathrm{CuCl}_{2}, \mathrm{NiCl}_{2}, \mathrm{Na}_{2} \mathrm{MoO}_{4}, \mathrm{Na}_{2} \mathrm{SeO}_{3}$, and $\mathrm{H}_{3} \mathrm{BO}_{3}$ in $60 \mathrm{mM} \mathrm{HCl}$ ). Cells were lysed in $50 \mathrm{mM}$ Tris pH 7.5, 500 $\mathrm{mM} \mathrm{NaCl}, 10 \mathrm{mM}$ imidazole, and $2 \mathrm{mM} \beta$-mercaptoethanol using a microfluidizer from Microfluidics. For Gsp1 purifications, the lysis buffer was also supplemented with $10 \mathrm{mM}$ $\mathrm{MgCl}_{2}$. The His-tagged proteins were purified on Ni-NTA resin (Thermo Scientific \#88222) and washed into a buffer containing $50 \mathrm{mM}$ Tris (pH 7.5) and $100 \mathrm{mM} \mathrm{NaCl}$, with 5 $\mathrm{mM} \mathrm{MgCl} 2$ for Gsp1 proteins. The N-terminal His-tag was digested at room temperature overnight using up to $12 \mathrm{NIH}$ Units per $\mathrm{mL}$ of bovine thrombin (Sigma-Aldrich T4648$10 \mathrm{KU})$. Proteins were then purified using size exclusion chromatography (HiLoad 26/600 Superdex 200 pg column from GE Healthcare), and purity was confirmed to be at least $90 \%$ by SDS polyacrylamide gel electrophoresis. Samples were concentrated on $10 \mathrm{kDa}$ spin filter columns (Amicon Catalog \# UFC901024) into storage buffer (50 mM Tris pH 7.5, 150 $\mathrm{mM} \mathrm{NaCl}, 1 \mathrm{mM}$ Dithiothreitol). Storage buffer for Gsp1 proteins was supplemented with $5 \mathrm{mM} \mathrm{MgCl} 2 .$. Protein concentrations were confirmed by measuring at 10-50x dilution using a Nanodrop (ThermoScientific). The extinction coefficient at $280 \mathrm{~nm}$ used for nucleotide (GDP or GTP) bound Gsp1 was 37675 M-1 cm-1, as described in (55). The ratio of absorbance at $260 \mathrm{~nm}$ and $280 \mathrm{~nm}$ for purified Gsp1 bound to GDP was 0.76. Extinction coefficients for other proteins were estimated based on their primary protein sequence using the ProtParam tool. Concentrated proteins were flash-frozen and stored at $-80^{\circ} \mathrm{C}$.

In our hands every attempt to purify the $S$. cerevisiae homologue of GAP (Rna1, Uniprot P11745) from E. coli yielded a protein that eluted in the void volume on the Sephadex 200 size exclusion column, indicating that the protein is forming soluble higher-order oligomers. We were, however, successful in purifying the S. pombe homologue of GAP (Rna1, Uniprot P41391) as a monomer of high purity as described above. S. pombe and S. cerevisiae Rnal proteins have an overall $43 \%$ sequence identity and $72 \%$ sequence similarity. The X-ray crystal structure of Ran GTPase and its GAP used in our analyses is a co-complex structure of the S. pombe homolog of Rna1 (PDB: 15kd), human RAN, and human RANBP1 (Supplementary File 1 Table 1). We used the purified $S$. pombe homolog of Rnal in all of our GTP hydrolysis kinetic experiments.

Circular dichroism (CD) spectroscopy of protein thermostability. Samples for CD analysis were prepared at approximately $2 \mu \mathrm{M}$ Gsp1 in $2 \mathrm{mM}$ HEPES $\mathrm{pH} 7.5,5 \mathrm{mM}$ $\mathrm{NaCl}, 200 \mu \mathrm{M} \mathrm{MgCl}_{2}$, and $50 \mu \mathrm{M}$ Dithiothreitol. CD spectra were recorded at $25^{\circ} \mathrm{C}$ using $2 \mathrm{~mm}$ cuvettes (Starna, 21-Q-2) in a JASCO J-710 CD-spectrometer (Serial \#9079119). The bandwidth was $2 \mathrm{~nm}$, rate of scanning $20 \mathrm{~nm} / \mathrm{min}$, data pitch $0.2 \mathrm{~nm}$, and response time $8 \mathrm{~s}$. Each CD spectrum represents the accumulation of 5 scans. Buffer spectra were subtracted from the sample spectra using the Spectra Manager software Version 1.53.01 from JASCO Corporation. Temperature melts were performed from $25^{\circ} \mathrm{C}-95^{\circ} \mathrm{C}$, monitoring at $210 \mathrm{~nm}$, using a data pitch of $0.5^{\circ} \mathrm{C}$ and a temperature slope of $1^{\circ} \mathrm{C}$ per minute. As all thermal melts of wild type and mutant Gsp1 proteins were irreversible, only apparent Tm was estimated (Supplementary File 1 Fig. 11) and is reported in Supplementary File 1 Table 9.

GTP loading of Gsp1. Gsp1 variants for GTPase assays as well as for ${ }^{31} \mathrm{P}$ NMR spectroscopy were first loaded with GTP by incubation in the presence of 20 -fold excess GTP (Guanosine 5-Triphosphate, Disodium Salt, CAT \# 371701, Calbiochem) in $50 \mathrm{mM}$ Tris $\mathrm{HCl} \mathrm{pH} 7.5,100 \mathrm{mM} \mathrm{NaCl}, 5$ $\mathrm{mM} \mathrm{MgCl} 2$. Exchange of GDP for GTP was initiated by the addition of $10 \mathrm{mM}$ EDTA. Reactions were incubated for 3 hours at $4^{\circ} \mathrm{C}$ and stopped by addition of $1 \mathrm{M} \mathrm{MgCl}_{2}$ to a final concentration of $20 \mathrm{mM} \mathrm{MgCl} 2$ to quench the EDTA. GTP-loaded protein was buffer exchanged into either NMR buffer or the GTPase assay buffer using NAP-5 Sephadex G25 DNA Grade columns (GE Healthcare \# 17085301).

Reverse phase high performance liquid chromatography (HPLC). Analysis of bound nucleotide was performed using reverse-phase chromatography as previously described (55) using a C18 column (HAISIL TS Targa C18, particle size $5 \mu \mathrm{m}$, pore size $120 \AA$, dimensions 150 x $4.6 \mathrm{~mm}$, Higgins Analytical \# TS-1546-C185). The column was preceded by a precolumn filter (The Nest Group, Inc, Part \# UA318, requires $0.5 \mu \mathrm{m}$ frits, Part \# UA102) and a C18 guard column (HAICart SS Cartridge Column, HAISIL Targa C18, $3.2 \times 20 \mathrm{~mm}, 5 \mu \mathrm{m}, 120 \AA$ A Higgins Analytical \# TF-0232C185, requires a Guard Holder Kit, Higgins Analytical \# HK-GUARD-FF). To prepare the nucleotide for analysis, a 
Gsp1 sample was first diluted to a concentration of $25-30$ $\mu \mathrm{M}$ and a volume of $40 \mu \mathrm{l}$. The protein was denatured by addition of $2.5 \mu \mathrm{l}$ of $10 \%$ perchloric acid $\left(\mathrm{HClO}_{4}\right)$. The $\mathrm{pH}$ was raised by addition of $1.75 \mu \mathrm{l} 4 \mathrm{M}$ sodium acetate $\left(\mathrm{CH}_{3} \mathrm{COONa}\right) \mathrm{pH}$ 4.0. The nucleotide was separated from the precipitated protein before application to the column by spinning at $20,000 \mathrm{x}$ g for 20 minutes. $30 \mu \mathrm{l}$ of supernatant was withdrawn and mixed 1:1 with reverse-phase buffer (10

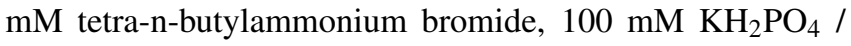
$\mathrm{K}_{2} \mathrm{HPO}_{4}$, pH 6.5, $0.2 \mathrm{mM} \mathrm{NaN}_{3}$ ). $20 \mu \mathrm{l}$ of sample was injected onto the equilibrated column, and was run isocratically in $92.5 \%$ reverse-phase buffer, $7.5 \%$ acetonitrile at a flow rate of $1 \mathrm{ml} / \mathrm{min}$ for $35 \mathrm{~min}$ ( 20 column volumes). Nucleotide retention was measured by monitoring absorbance at both $254 \mathrm{~nm}$ and $280 \mathrm{~nm}$. Example HPLC reverse phase chromatogram of GTP-loaded wild type Gsp1 is shown in Supplementary File 1 Fig. 12.

NMR Spectroscopy. Gsp1 samples for ${ }^{31} \mathrm{P}$ NMR spectroscopy were first loaded with GTP as described above, and buffer exchanged into NMR Buffer $\left(\mathrm{D}_{2} \mathrm{O}\right.$ with $50 \mathrm{mM}$ Tris$\mathrm{HCl} \mathrm{pH} \mathrm{7.4,} 5 \mathrm{mM} \mathrm{MgCl}_{2}, 2 \mathrm{mM}$ Dithiothreitol). Final sample concentrations were between $250 \mu \mathrm{M}$ and $2 \mathrm{mM}$, and 400 ul of sample was loaded into $5 \mathrm{~mm}$ Shigemi advanced microtubes matched to $\mathrm{D}_{2} \mathrm{O}$ (BMS-005TB; Shigemi Co. Ltd, Tokyo, Japan.). ${ }^{31} \mathrm{P}$ NMR experiments were performed on a Bruker Avance III $600 \mathrm{MHz}$ NMR spectrometer with a 5 $\mathrm{mm}$ BBFO Z-gradient Probe. Spectra were acquired and processed with the Bruker TopSpin software (version 4.0.3). Indirect chemical shift referencing for ${ }^{31} \mathrm{P}$ to DSS (2 mM Sucrose, $0.5 \mathrm{mM}$ DSS, $2 \mathrm{mM} \mathrm{NaN}_{3}$ in $90 \% \mathrm{H}_{2} \mathrm{O}+10 \% \mathrm{D}_{2} \mathrm{O}$; water-suppression standard) was done using the IUPAC-IUB recommended ratios (56). Spectra were recorded at $25^{\circ} \mathrm{C}$ using the pulse and acquire program $\mathrm{zg}$ (TopSpin 3.6.0), with an acquisition time of 280 milliseconds, a recycle delay of 3.84 seconds, and a $65^{\circ}$ hard pulse. 4,096 complex points were acquired over the course of 4,096 scans and a total acquisition time of 4.75 hours. Spectra were zero-filled once and multiplied with an exponential window function (EM) with a line-broadening of $6 \mathrm{~Hz}(\mathrm{LB}=6)$ prior to Fourier transformation. Peaks were integrated using the auto-integrate function in TopSpin 4.0.7, and peak areas were referenced to the bound GTP- $\beta$ peak of each spectrum. Values for the fraction of each variant in state 2 were computed by taking the area of the GTP- $\gamma 2$ peak and dividing by the sum of the two GTP- $\gamma$ peak areas.

Kinetic measurements of GTP hydrolysis. Kinetic parameters of the GTP hydrolysis reaction were determined using a protocol similar to one previously described (57). Gsp1 samples for GTP hydrolysis kinetic assays were first loaded with GTP as described above. GTP hydrolysis was monitored by measuring fluorescence of the E. coli phosphate-binding protein labeled with 7-Diethylamino-3[N-(2-maleimidoethyl)carbamoyl]coumarin (MDCC) (phosphate sensor, CAT \# PV4406, Thermo Fisher) upon binding of the free phosphate GTP hydrolysis product (excitation at $425 \mathrm{~nm}$, emission at $457 \mathrm{~nm}$ ). All experiments were performed in GTPase assay buffer (40 mM HEPES pH 7.5,
$100 \mathrm{mM} \mathrm{NaCl}, 4 \mathrm{mM} \mathrm{MgCl} 2,1 \mathrm{mM}$ Dithiothreitol) at $30^{\circ} \mathrm{C}$ in $100 \mu \mathrm{l}$ reaction volume using a Synergy $\mathrm{H} 1$ plate reader from BioTek, using Corning 3881 96-well half-area clearbottom non-binding surface plates. The phosphate sensor at $20 \mu \mathrm{M}$ and $50 \mu \mathrm{M}$ concentrations was calibrated with a range of concentrations of $\mathrm{K}_{2} \mathrm{HPO}_{4}$ using only the data in the linear range. For most mutants a concentration of $1 \mathrm{nM}$ GAP (SpRna1, Rnal from S. pombe) was used. In order to run the time courses to completion, for mutants with low $\mathrm{k}_{\mathrm{cat}} / \mathrm{K}_{\mathrm{m}}$ enzyme concentrations of 2-7 $\mathrm{nM}$ were used. Initially we collected time course data for all Gsp1 variants at approximately $8 \mu \mathrm{M}$ concentration of loaded Gsp1:GTP with $1 \mathrm{nM}$ GAP and $20 \mu \mathrm{M}$ phosphate sensor. Exact concentrations of loaded Gsp1:GTP were then estimated based on the plateau fluorescence and the sensor calibration parameters. Each time course was then fitted to an integrated Michaelis Menten equation using a Lambert $\mathrm{W}$ function, as previously described (58). We analysed the data with the custom-made software DELA written by David Lambright from University of Massachusetts Medical School and generously made available to us as freeware. If the estimated $\mathrm{K}_{\mathrm{m}}$ was higher than $1 \mu \mathrm{M}$, we repeated the time course kinetic experiments with higher concentration of Gsp1:GTP of approximately tenfold above the $K_{m}$. The Michaelis Menten $k_{c a t}$ and $K_{m}$ parameters and their standard deviations were calculated from at least 2-9 technical replicates from 1-4 independently GTPloaded Gsp1 samples (Supplementary File 1 Table 6). For each individual GAP-mediated GTP hydrolysis experiment, a control experiment with the same concentration of GTPloaded Gsp1 and the same concentration of sensor, but without added GAP was ran in parallel. The first 100 seconds of these data were used to determine the baseline fluorescence, and the rest of the data were linearly fit to estimate intrinsic GTP hydrolysis rate (Supplementary File 1 Table 8). Example data and integrated Michaelis-Menten fits are show in Supplementary File 1 Fig. 2.

Kinetic measurements of Srm1 mediated nucleotide exchange. Kinetic parameters of GEF mediated nucleotide exchange were determined using a fluorescence resonance energy transfer (FRET) based protocol (59). Each Gsp1 variant was purified as a Gsp1:GDP complex, as confirmed by reverse phase chromatography. Nucleotide exchange from GDP to mant-GTP (2'-(or-3')-O-(N-Methylanthraniloyl) Guanosine 5-Triphosphate, CAT \# NU-206L, Jena Biosciences) was monitored by measuring a decrease in intrinsic Gsp1 tryptophan fluorescence (295 nm excitation, 335 $\mathrm{nm}$ detection) due to FRET upon binding of the mant group. Each time course was measured in GEF assay buffer (40 $\mathrm{mM}$ HEPES pH 7.5, $100 \mathrm{mM} \mathrm{NaCl}, 4 \mathrm{mM} \mathrm{MgCl} 2,1 \mathrm{mM}$ Dithiothreitol) with excess of mant-GTP. For most variants of Gsp1 we measured time courses at Gsp1:GDP concentrations ranging from 0.25 to $12 \mu \mathrm{M}$ with an excess mant-GTP concentration of $200 \mu \mathrm{M}$. For Gsp1 variants with high $\mathrm{K}_{\mathrm{m}}$ values that had to be measured at concentrations of up to 200 $\mu \mathrm{M}$ we used an excess of $1000 \mu \mathrm{M}$ mant-GTP. All kinetic measurements were done at $30^{\circ} \mathrm{C}$ in $100 \mu \mathrm{l}$ reaction volume using $5 \mathrm{nM}$ GEF ( $\Delta 1-27 \mathrm{Srm} 1)$, except for higher concen- 
trations of the mutants with high $\mathrm{K}_{\mathrm{m}}$ values that were measured at $20 \mathrm{nM} \mathrm{GEF}$. Data were collected in a Synergy H1 plate reader from BioTek, using Corning 3686 96-well halfarea non-binding surface plates. For low concentrations of Gsp1:GDP the time course data were fit to a combination of two exponential decays:

$$
Y=\operatorname{span}_{1} e^{k_{\text {nuc.exch. Time }}}+\operatorname{span}_{2} e^{k_{b g} \text { Time }}+\text { plateau }
$$

where $\mathrm{k}_{\text {nuc. exch }}$ is the rate constant of the GDP to mant-GTP exchange, $k_{b g}$ is the rate constant of background decay due to photo-bleaching, and $\operatorname{span}_{1}$ and $\operatorname{span}_{2}$ are the fluorescence amplitudes for the two processes. For high concentrations of substrate, or for mutants with very low rates, the initial velocity was determined by a linear fit to the initial $10-20 \%$ of the data. The kinetic parameters of the nucleotide exchange were determined by fitting a Michaelis-Menten equation to an average of 38 data points (ranging from 17 to 91) per Gsp1 point mutant for a range of substrate concentrations from [Gsp1:GDP] $=0.25 \mu \mathrm{M}$ to [Gsp1:GDP] $\gg \mathrm{K}_{\mathrm{m}}$. Example data and Michaelis-Menten fits are shown in Supplementary File 1 Fig. 3. Michaelis-Menten $\mathrm{k}_{\mathrm{cat}}$ and $\mathrm{K}_{\mathrm{m}}$ parameters for GEF-mediated nucleotide exchange are provided in Supplementary File 1 Table 7. The errors of the $\mathrm{k}_{\text {cat }}$ and the $\mathrm{K}_{\mathrm{m}}$ parameters were determined from the standard error of the exponential fit of the Michaelis-Menten equation to the data. The error of the catalytic efficiency $\left(\mathrm{k}_{\mathrm{cat}} / \mathrm{K}_{\mathrm{m}}\right)$ was calculated by adding the standard errors of the individual parameters and normalizing it for the values of the parameters

$$
\frac{k_{\text {cat }}}{K_{m}} \sqrt{\left(\frac{\text { std.error }\left(k_{c a t}\right)}{k_{c a t}}\right)^{2}+\left(\frac{\text { std.error }\left(K_{m}\right)}{K_{m}}\right)^{2}} .
$$

All the custom written code for fitting and analysis of kinetics data is provided in the accompanying kinetics GitHub repository.

\section{Acknowledgements}

We thank Roxana Ordonez for contributions to the design of mutations; Cristina Melero, Deborah Jeon, Shivani Mathur, Raina Danbi Kim, and Kale Kundert for technical help; Maru Jaime Garza for contributions to the conformational analysis by NMR; Colm Ryan for advice on E-MAP analysis; David Lambright for guidance and software to analyze kinetics of GAP-mediated hydrolysis; and Dave Agard, Geeta Narlikar, James Fraser and Janet M. Thornton for stimulating discussions; Ricardo Henriques and his lab for the preprint template. This work was supported by a grant from the National Institutes of Health (R01-GM117189) to T.K., and a Sir Henry Wellcome Postdoctoral Fellowship (101614/Z/13/Z) to T.P. C.J.P.M. is a UCSF Discovery Fellow. T.K. is a Chan Zuckerberg Biohub investigator.

\section{Author Contributions}

T.P., C.J.P.M., N.J.K. and T.K. identified and developed the core questions. T.P. and C.J.P.M. performed the bulk of the experiments and data analysis. J.X. and T.P. performed the
E-MAP screens. G.M.J. performed the pull-down experiments. D.L.S. and R.K. performed the MS experiments and together with T.P. analyzed the data. N.O. contributed to design of Gsp1 mutants. H.B. contributed to E-MAP analysis. M.J.S.K. suggested the NMR studies. C.J.P.M. and M.J.S.K. performed the NMR experiments and analyzed the data. Y.Z. performed the Western blot experiments. T.P., C.J.P.M., and Y.Z. prepared the proteins. T.P. performed the kinetics experiments. T.P., C.J.P.M. and T.K. wrote the manuscript with contributions from the other authors. N.J.K. and T.K. oversaw the project.

\section{Data Availability}

The mass spectrometry proteomics data have been deposited to the PRIDE proteomics data repository with the dataset identifier PXD016338 and are available as Supplementary Tables. Raw biophysics data (cycle kinetics, CD, and NMR), and E-MAP S-scores, scaled SGA (44) scores, and their correlations are available from a GitHub repository All other data that support the findings of this study are available within the paper and its Supplementary Files.

\section{Code Availability}

Custom written R and Python scripts are available without restrictions from a asp1 manuscript GitHub repository

\section{Competing Interests}

The authors declare no competing interests.

\section{References}

1. D Eisenberg, E M Marcotte, I Xenarios, and T O Yeates. Protein function in the post-genomic era. Nature, 405(6788):823-826, June 2000.

2. Ian A Mellis and Arjun Raj. Half dozen of one, six billion of the other: What can small- and large-scale molecular systems biology learn from one another? Genome Research, 25(10): 1466-1472, October 2015.

3. Marija Buljan, Peter Blattmann, Ruedi Aebersold, and Michael Boutros. Systematic characterization of pan-cancer mutation clusters. Molecular Systems Biology, 14(3):e7974, March 2018.

4. Benjamin Schuster-Böckler and Alex Bateman. Protein interactions in human genetic diseases. Genome Biology, 9(1):R9, 2008.

5. Emmanuel D Levy. A simple definition of structural regions in proteins and its use in analyzing interface evolution. Journal Of Molecular Biology, 403(4):660-670, November 2010.

6. A Goldbeter and D E Koshland. An amplified sensitivity arising from covalent modification in biological systems. Proceedings of the National Academy of Sciences, 78(11):6840-6844, November 1981

7. D Pincus, I Letunic, P Bork, and W A Lim. Evolution of the phospho-tyrosine signaling machinery in premetazoan lineages. Proceedings of the National Academy of Sciences, 105(28):9680-9684, July 2008

8. Mary Dasso. The Ran GTPase: theme and variations. Current Biology, 12(14):R502-8, July 2002.

9. F R Bischoff and $H$ Ponstingl. Ran Regulation by Ran GEF and Ran GAP. In M G Rush and P D'Eustachio, editors, The Small GTPase Ran, pages 163-176. Springer, Boston, MA, Boston, August 2001.

10. M. S. Moore and G Blobel. The GTP-binding protein Ran/TC4 is required for protein import into the nucleus. Nature, 365(6447):661-663, October 1993.

11. Murray Stewart. Molecular mechanism of the nuclear protein import cycle. Nature Reviews Molecular Cell Biology, 8(3):195-208, March 2007.

12. Alwin Köhler and Ed Hurt. Exporting RNA from the nucleus to the cytoplasm. Nature Reviews Molecular Cell Biology, 8(10):761-773, October 2007.

13. Mildred Delaleau and Katherine $L$ B Borden. Multiple Export Mechanisms for mRNAs. Cells, 4(3):452, September 2015.

14. Alexei Arnaoutov and Mary Dasso. The Ran GTPase regulates kinetochore function. Developmental Cell, 5(1):99-111, July 2003.

15. M Ren, A Villamarin, A Shih, E Coutavas, M S Moore, M LoCurcio, V Clarke, J D Oppenheim, P D'Eustachio, and M G Rush. Separate domains of the Ran GTPase interact with different factors to regulate nuclear protein import and RNA processing. Molecular and Cellular Biology, 15(4):2117-2124, April 1995. 
bioRxiv preprint doi: https://doi.org/10.1101/2020.01.04.893909; this version posted January 6, 2020. The copyright holder for this preprint

(which was not certified by peer review) is the author/funder, who has granted bioRxiv a license to display the preprint in perpetuity. It is made available under aCC-BY-NC-ND 4.0 International license.

16. M Hetzer, D Bilbao-Cortés, T C Walther, O J Gruss, and I W Mattaj. GTP hydrolysis by Ran is required for nuclear envelope assembly. Molecular Cell, 5(6):1013-1024, June 2000.

17. Ana Maria Rojas, Gloria Fuentes, Antonio Rausell, and Alfonso Valencia. The Ras protein superfamily: evolutionary tree and role of conserved amino acids. The Journal of Cell Biology, 196(2):189-201, January 2012.

18. Hannes Braberg, Huiyan Jin, Erica A Moehle, Yujia A Chan, Shuyi Wang, Michael Shales, Joris J Benschop, John H Morris, Chenxi Quu, Fuqu Hu, Leung K Tang, James S Fraser, Frank C P Holstege, Philip Hieter, Christine Guthrie, Craig D Kaplan, and Nevan J Krogan. From structure to systems: high-resolution, quantitative genetic analysis of RNA polymerase II. Cell, 154(4):775-788, August 2013.

19. Maya Schuldiner, Sean R Collins, Natalie J Thompson, Vladimir Denic, Arunashree Bhamidipati, Thanuja Punna, Jan Ihmels, Brenda Andrews, Charles Boone, Jack F Greenblatt, Jonathan $\mathrm{S}$ Weissman, and Nevan J Krogan. Exploration of the function and organization of the yeast early secretory pathway through an epistatic miniarray profile. Cell, 123 (3):507-519, November 2005.

20. M Costanzo, B VanderSluis, E N Koch, A Baryshnikova, C Pons, G Tan, W Wang, M Usaj, J Hanchard, S D Lee, V Pelechano, E B Styles, M Billmann, J van Leeuwen, N van Dyk, ZY Lin, E Kuzmin, J Nelson, J S Piotrowski, T Srikumar, S Bahr, Y Chen, R Deshpande, C F Kurat, S C Li, Z Li, M M Usaj, H Okada, N Pascoe, B J San Luis, S Sharifpoor, E Shuteriqi, S W Simpkins, J Snider, H G Suresh, Y Tan, H Zhu, N Malod-Dognin, V Janjic, N Przulj, O G Troyanskaya, I Stagljar, T Xia, Y Ohya, A C Gingras, B Raught, M Boutros, L M Steinmetz, C L Moore, A P Rosebrock, A A Caudy, C L Myers, B Andrews, and C Boone. A global genetic interaction network maps a wiring diagram of cellular function. Science (New York, NY), 353(6306):aaf1420-aaf1420, September 2016

21. Sean R Collins, Maya Schuldiner, Nevan J Krogan, and Jonathan S Weissman. A strategy for extracting and analyzing large-scale quantitative epistatic interaction data. Genome Biology, 7(7):R63, 2006

22. M Geyer, R Assheuer, C Klebe, J Kuhlmann, J Becker, A Wittinghofer, and H R Kalbitzer. Conformational States of the Nuclear GTP-Binding Protein Ran and Its Complexes with the Exchange Factor RCC1 and the Effector Protein RanBP1. . Biochemistry, 38(35):1125011260, August 1999.

23. M Geyer, T Schweins, C Herrmann, T Prisner, A Wittinghofer, and H R Kalbitzer. Conformational transitions in p21ras and in its complexes with the effector protein Raf-RBD and the GTPase activating protein GAP. Biochemistry, 35(32):10308-10320, August 1996.

24. Jude Canon, Karen Rex, Anne Y Saiki, Christopher Mohr, Keegan Cooke, Dhanashri Bagal, Kevin Gaida, Tyler Holt, Charles G Knutson, Neelima Koppada, Brian A Lanman, Jonathan Werner, Aaron S Rapaport, Tisha San Miguel, Roberto Ortiz, Tao Osgood, Ji-Rong Sun, Xiaochun Zhu, John D McCarter, Laurie P Volak, Brett E Houk, Marwan G Fakih, Bert H O'Neil, Timothy J Price, Gerald S Falchook, Jayesh Desai, James Kuo, Ramaswamy Govindan, David S Hong, Wenjun Ouyang, Haby Henary, Tara Arvedson, Victor J Cee, and J Russell Lipford. The clinical KRAS(G12C) inhibitor AMG 510 drives anti-tumour immunity. $\mathrm{Na}$ ture, 56:779-21, October 2019.

25. Dirk Kessler, Michael Gmachl, Andreas Mantoulidis, Laetitia J Martin, Andreas Zoephel, Moriz Mayer, Andreas Gollner, David Covini, Silke Fischer, Thomas Gerstberger, Teresa Gmaschitz, Craig Goodwin, Peter Greb, Daniela Häring, Wolfgang Hela, Johann Hoffmann, Jale Karolyi-Oezguer, Petr Knesl, Stefan Kornigg, Manfred Koegl, Roland Kousek, Lyne Lamarre, Franziska Moser, Silvia Munico-Martinez, Christoph Peinsipp, Jason Phan, Jörg Rinnenthal, Jiqing Sai, Christian Salamon, Yvonne Scherbantin, Katharina Schipany, Renate Schnitzer, Andreas Schrenk, Bernadette Sharps, Gabriella Siszler, Qi Sun, Alex Waterson, Bernhard Wolkerstorfer, Markus Zeeb, Mark Pearson, Stephen W Fesik, and Darryl B McConnell. Drugging an undruggable pocket on KRAS. Proceedings of the National Academy of Sciences, 116(32):15823-15829, August 2019.

26. Jonathan M Ostrem, Ulf Peters, Martin L Sos, James A Wells, and Kevan M Shokat. KRas(G12C) inhibitors allosterically control GTP affinity and effector interactions. Nature, 503(7477):548-551, November 2013.

27. Chunaram Choudhary, Chanchal Kumar, Florian Gnad, Michael L Nielsen, Michael Rehman, Tobias C Walther, Jesper V Olsen, and Matthias Mann. Lysine acetylation targets protein complexes and co-regulates major cellular functions. Science (New York, NY), 325(5942):834-840, August 2009.

28. Susanne de Boor, Philipp Knyphausen, Nora Kuhlmann, Sarah Wroblowski, Julian Brenig, Lukas Scislowski, Linda Baldus, Hendrik Nolte, Marcus Krüger, and Michael Lammers. Small GTP-binding protein Ran is regulated by posttranslational lysine acetylation. Proceedings of the National Academy of Sciences, 112(28):E3679-88, July 2015.

29. M G Rush, G Drivas, and P D'Eustachio. The small nuclear GTPase Ran: how much does it run? BioEssays : news and reviews in molecular, cellular and developmental biology, 18 (2):103-112, February 1996

30. F R Bischoff, H Krebber, E Smirnova, W Dong, and H Ponstingl. Co-activation of RanGTPase and inhibition of GTP dissociation by Ran-GTP binding protein RanBP1. The EMBO Journal, 14(4):705-715, February 1995.

31. Michael J Seewald, Carolin Körner, Alfred Wittinghofer, and Ingrid R Vetter. RanGAP mediates GTP hydrolysis without an arginine finger. Nature, 415(6872):662-666, February 2002.

32. E Besray Unal, Christina Kiel, Hannah Benisty, Andrew Campbell, Karen Pickering, Nils Blüthgen, Owen J Sansom, and Luis Serrano. Systems level expression correlation of Ras GTPase regulators. Cell Communication and Signaling : CCS, 16(1):46, August 2018.

33. Dirk Görlich, Michael J Seewald, and Katharina Ribbeck. Characterization of Ran-driven cargo transport and the RanGTPase system by kinetic measurements and computer simulation. The EMBO Journal, 22(5):1088-1100, March 2003.

34. Francis A Barr. Review series: Rab GTPases and membrane identity: causal or inconsequential? The Journal of cell Biology, 202(2):191-199, July 2013.

35. Caleb J Bashor, Andrew A Horwitz, Sergio G Peisajovich, and Wendell A Lim. Rewiring cells: synthetic biology as a tool to interrogate the organizational principles of living systems. Annual Review of Biophysics, 39:515-537, 2010.

36. Matthew Z Tien, Austin G Meyer, Dariya K Sydykova, Stephanie J Spielman, and Claus O Wilke. Maximum allowed solvent accessibilites of residues in proteins. PLOS ONE, 8(11): e80635, 2013
37. Barry J Grant, Ana P C Rodrigues, Karim M EISawy, J Andrew McCammon, and Leo S D Caves. Bio3d: an R package for the comparative analysis of protein structures. Bioinformatics (Oxford, England), 22(21):2695-2696, November 2006.

38. Sean R Collins, Assen Roguev, and Nevan J Krogan. Quantitative genetic interaction mapping using the E-MAP approach. Methods in Enzymology, 470:205-231, 2010.

39. Guri Giaever, Angela M Chu, Li Ni, Carla Connelly, Linda Riles, Steeve Véronneau, Sally Dow, Ankuta Lucau-Danila, Keith Anderson, Bruno André, Adam P Arkin, Anna Astromoff, Mohamed El-Bakkoury, Rhonda Bangham, Rocio Benito, Sophie Brachat, Stefano Campanaro, Matt Curtiss, Karen Davis, Adam Deutschbauer, Karl-Dieter Entian, Patrick Flaherty, Francoise Foury, David J Garfinkel, Mark Gerstein, Deanna Gotte, Ulrich Güldener, Johannes H Hegemann, Svenja Hempel, Zelek Herman, Daniel F Jaramillo, Diane E Kelly, Steven L Kelly, Peter Kötter, Darlene LaBonte, David C Lamb, Ning Lan, Hong Liang, Hong Liao, Lucy Liu, Chuanyun Luo, Marc Lussier, Rong Mao, Patrice Ménard, Siew Loon Ooi, Jose L Revuelta, Christopher J Roberts, Matthias Rose, Petra Ross-Macdonald, Bart Scherens, Greg Schimmack, Brenda Shafer, Daniel D Shoemaker, Sharon SookhaiMahadeo, Reginald K Storms, Jeffrey N Strathern, Giorgio Valle, Marleen Voet, Guido Volckaert, Ching-yun Wang, Teresa R Ward, Julie Wilhelmy, Elizabeth A Winzeler, Yonghong Yang, Grace Yen, Elaine Youngman, Kexin Yu, Howard Bussey, Jef D Boeke, Michael Snyder, Peter Philippsen, Ronald W Davis, and Mark Johnston. Functional profiling of the Saccharomyces cerevisiae genome. Nature, 418(6896):387-391, July 2002.

40. David K Breslow, Dale M Cameron, Sean R Collins, Maya Schuldiner, Jacob StewartOrnstein, Heather W Newman, Sigurd Braun, Hiten D Madhani, Nevan J Krogan, and Jonathan S Weissman. A comprehensive strategy enabling high-resolution functional analysis of the yeast genome. Nature Methods, 5(8):711-718, August 2008.

41. Suzanne Hoppins, Sean R Collins, Ann Cassidy-Stone, Eric Hummel, Rachel M Devay, Laura L Lackner, Benedikt Westermann, Maya Schuldiner, Jonathan S Weissman, and Jodi Nunnari. A mitochondrial-focused genetic interaction map reveals a scaffold-like complex required for inner membrane organization in mitochondria. The Journal of cell Biology, 195 (2):323-340, October 2011

42. J M Cherry, C Adler, C Ball, S A Chervitz, S S Dwight, E T Hester, Y Jia, G Juvik, T Roe, M Schroeder, S Weng, and D Botstein. SGD: Saccharomyces Genome Database. Nucleic Acids Research, 26(1):73-79, January 1998.

43. Zuguang Gu, Roland Eils, and Matthias Schlesner. Complex heatmaps reveal patterns and correlations in multidimensional genomic data. Bioinformatics (Oxford, England), 32(18): 2847-2849, September 2016

44. Matej Usaj, Yizhao Tan, Wen Wang, Benjamin VanderSluis, Albert Zou, Chad L Myers, Michael Costanzo, Brenda Andrews, and Charles Boone. TheCellMap.org: A WebAccessible Database for Visualizing and Mining the Global Yeast Genetic Interaction Network. G3 (Bethesda, Md.), 7(5):1539-1549, May 2017.

45. Colm J Ryan, Assen Roguev, Kristin Patrick, Jiewei Xu, Harlizawati Jahari, Zongtian Tong, Pedro Beltrao, Michael Shales, Hong Qu, Sean R Collins, Joseph I Kliegman, Lingli Jiang, Dwight Kuo, Elena Tosti, Hyun-Soo Kim, Winfried Edelmann, Michael-Christopher Keogh, Derek Greene, Chao Tang, Pádraig Cunningham, Kevan M Shokat, Gerard Cagney, J Peter Svensson, Christine Guthrie, Peter J Espenshade, Trey Ideker, and Nevan J Krogan. Hierarchical Modularity and the Evolution of Genetic Interactomes across Species. Molecular Cell, 46(5):691-704, June 2012.

46. Sean R Collins, Kyle M Miller, Nancy L Maas, Assen Roguev, Jeffrey Fillingham, Clement S Chu, Maya Schuldiner, Marinella Gebbia, Judith Recht, Michael Shales, Huiming Ding, Hong Xu, Junhong Han, Kristin Ingvarsdottir, Benjamin Cheng, Brenda Andrews, Charles Boone, Shelley L Berger, Phil Hieter, Zhiguo Zhang, Grant W Brown, C James Ingles, Andrew Emili, C David Allis, David P Toczyski, Jonathan S Weissman, Jack F Greenblatt, and Nevan J Krogan. Functional dissection of protein complexes involved in yeast chromosome biology using a genetic interaction map. Nature, 446(7137):806-810, April 2007.

47. Yoav Benjamini and Yosef Hochberg. Controlling the False Discovery Rate - a Practical and Powerful Approach to Multiple Testing. Journal of the Royal Statistical Society Series B-Statistical Methodology, 57(1):289-300, 1995.

48. Stefanie Jäger, Peter Cimermancic, Natali Gulbahce, Jeffrey R Johnson, Kathryn E McGovern, Starlynn C Clarke, Michael Shales, Gaelle Mercenne, Lars Pache, Kathy Li, Hilda Hernandez, Gwendolyn M Jang, Shoshannah L Roth, Eyal Akiva, John Marlett, Melanie Stephens, Iván D'Orso, Jason Fernandes, Marie Fahey, Cathal Mahon, Anthony J O'Donoghue, Aleksandar Todorovic, John H Morris, David A Maltby, Tom Alber, Gerard Cagney, Frederic D Bushman, John A Young, Sumit K Chanda, Wesley I Sundquist, Tanja Kortemme, Ryan D Hernandez, Charles S Craik, Alma Burlingame, Andrej Sali, Alan D Frankel, and Nevan J Krogan. Global landscape of HIV-human protein complexes. Nature, 481(7381):365-370, December 2011

49. Stefanie Jäger, Natali Gulbahce, Peter Cimermancic, Joshua Kane, Nanhai He, Seemay Chou, Iván D’Orso, Jason Fernandes, Gwendolyn Jang, Alan D Frankel, Tom Alber, Qiang Zhou, and Nevan J Krogan. Purification and characterization of HIV-human protein complexes. Methods (San Diego, Calif.), 53(1):13-19, January 2011

50. Jürgen Cox, Marco Y Hein, Christian A Luber, Igor Paron, Nagarjuna Nagaraj, and Matthias Mann. Accurate proteome-wide label-free quantification by delayed normalization and maximal peptide ratio extraction, termed MaxLFQ. Molecular \& Cellular Proteomics : MCP, 13 (9):2513-2526, September 2014.

51. Jürgen Cox and Matthias Mann. MaxQuant enables high peptide identification rates, individualized p.p.b.-range mass accuracies and proteome-wide protein quantification. Nature Biotechnology, 26(12):1367-1372, December 2008

52. Guoci Teo, Guomin Liu, Jianping Zhang, Alexey I Nesvizhskii, Anne-Claude Gingras, and Hyungwon Choi. SAINTexpress: improvements and additional features in Significance Analysis of INTeractome software. Journal of Proteomics, 100:37-43, April 2014.

53. Meena Choi, Ching-Yun Chang, Timothy Clough, Daniel Broudy, Trevor Killeen, Brendan MacLean, and Olga Vitek. MSstats: an R package for statistical analysis of quantitative mass spectrometry-based proteomic experiments. Bioinformatics (Oxford, England), 30 (17):2524-2526, September 2014

54. F William Studier. Protein production by auto-induction in high density shaking cultures. Protein expression and purification, 41(1):207-234, May 2005.

55. Susan J M Smith and Katrin Rittinger. Preparation of GTPases for Structural and Biophysical 
bioRxiv preprint doi: https://doi.org/10.1101/2020.01.04.893909; this version posted January 6, 2020. The copyright holder for this preprint

(which was not certified by peer review) is the author/funder, who has granted bioRxiv a license to display the preprint in perpetuity. It is made available under aCC-BY-NC-ND 4.0 International license.

Analysis. Methods in Molecular Biology (Clifton, N.J.), 189:013-024, June 2002.

56. John L Markley, Ad Bax, Yoji Arata, C W Hilbers, Robert Kaptein, Brian D Sykes, Peter E Wright, and Kurt Wüthrich. Recommendations for the presentation of NMR structures of proteins and nucleic acids - IUPAC-IUBMB-IUPAB Inter-Union Task Group on the Standardization of Data Bases of Protein and Nucleic Acid Structures Determined by NMR Spectroscopy. Journal of Biomolecular NMR, 12(1):1-23, 1998.

57. Ashwini K Mishra and David G Lambright. High-throughput assay for profiling the substrate specificity of Rab GTPase-activating proteins. Methods in Molecular Biology (Clifton, N.J.), 1298:47-60, 2015.

58. C T Goudar, J R Sonnad, and R G Duggleby. Parameter estimation using a direct solution of the integrated Michaelis-Menten equation. Biochimica et Biophysica Acta, 1429(2):377383, January 1999 .

59. C Klebe, F R Bischoff, H Ponstingl, and A Wittinghofer. Interaction of the nuclear GTPbinding protein Ran with its regulatory proteins RCC1 and RanGAP1. Biochemistry, 34(2): 639-647, January 1995. 
bioRxiv preprint doi: https://doi.org/10.1101/2020.01.04.893909; this version posted January 6, 2020. The copyright holder for this preprint (which was not certified by peer review) is the author/funder, who has granted bioRxiv a license to display the preprint in perpetuity. It is made available under aCC-BY-NC-ND 4.0 International license.

a

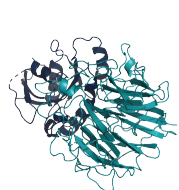

Srm1

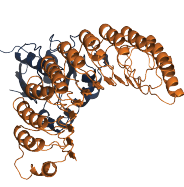

Rna1

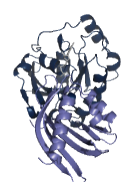

Ntf2

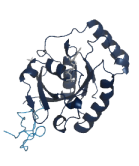

Nup1, Nup60

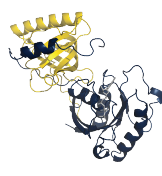

Yrb1

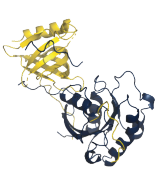

Yrb2

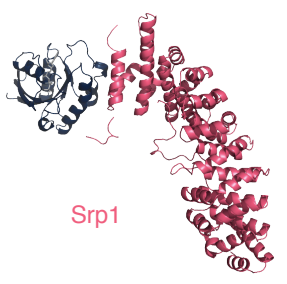

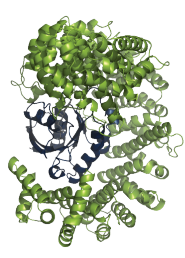

Kap95

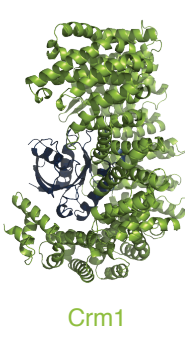

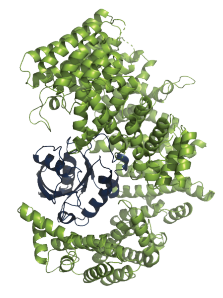

Los1

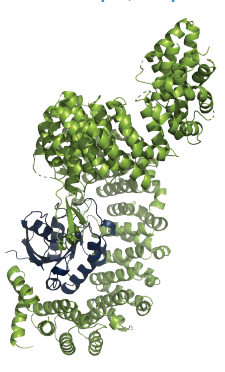

Pse1

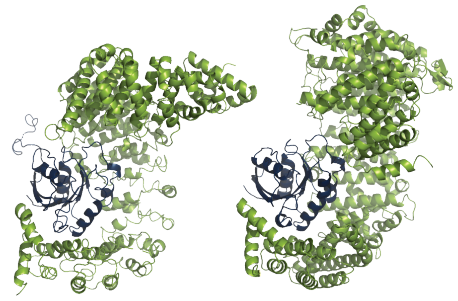

Kap104

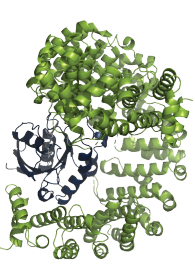

Cse1

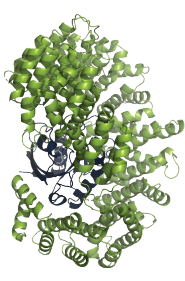

Mtr10

b

- mutated $\bigcirc$ not mutated

interface:

core

rim / support

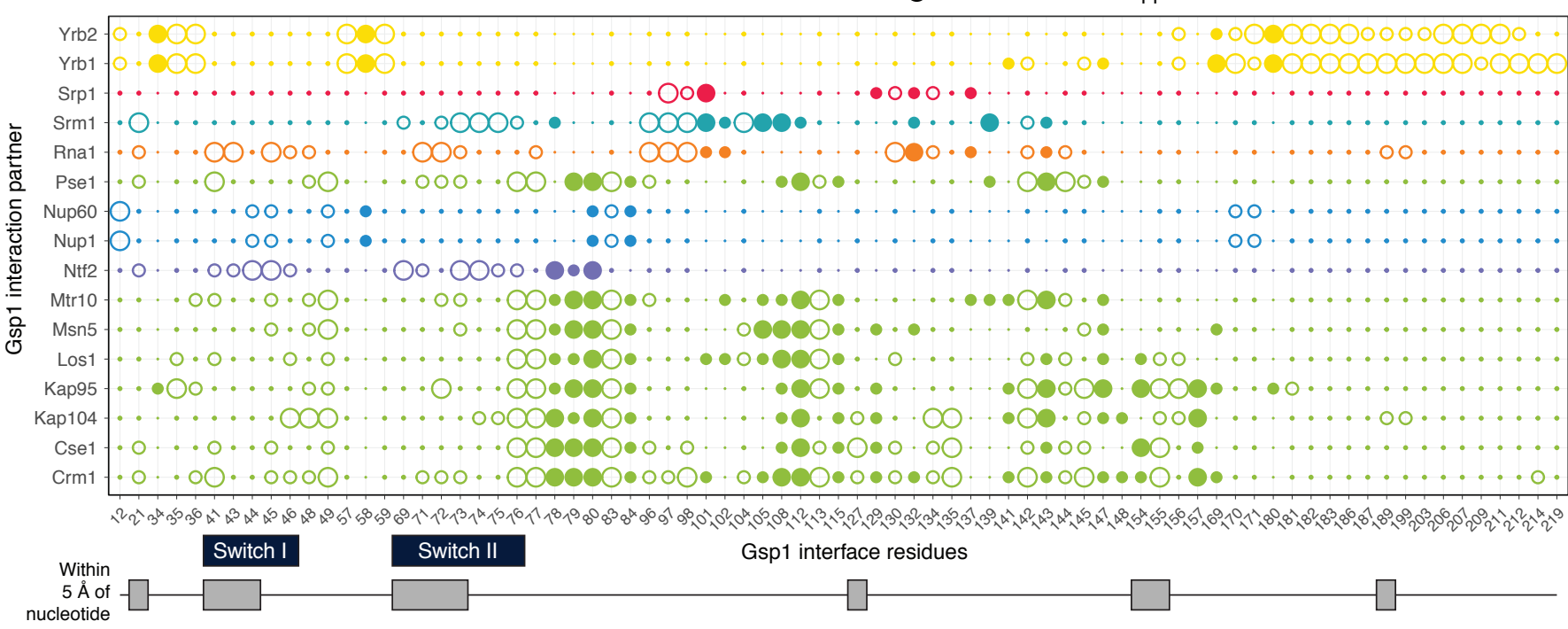

Fig. S1 Design of interface point mutations in S. cerevisiae Gsp1. a, Cartoon representation of co-complex structures of $S$. cerevisiae Gsp1 with indicated partners (or homologs): Srm1 (PDB: 1i2m), Rna1 (PDB: 1k5d), Ntf2 (PDB: 1a2k), Nup1/Nup60 (PDB: 3ch5), Yrb1 (PDB: 3m1i), Yrb2 (PDB: 3wyf), Srp1 (PDB: 1wa5), Kap95 (PDB: 2bku), Crm1 (PDB: 3m1i), Los1 (PDB: 3icq), Pse1(PDB: 3w3z), Kap104 (PDB: 1qbk), Msn5 (PDB: 3a6p), Cse1 (PDB: 1wa5), Mtr10 (PDB: 4ol0). Species and sequence identity to S. cerevisiae homologs for these structures are provided in Supplementary File 1 Table 1. b, Location of Gsp1 residues in partner interfaces. Interface positions (core, rim/support) were defined by the difference in relative surface accessible surface area ( $\Delta$ rASA) between monomer and complex (Methods, Supplementary File 1 Table 2). Residues within $5 \AA$ of the nucleotide, in the canonical P-loop, or in the switch I or II regions are indicated and were not mutated. Chosen Gsp1 point mutation substitutions are provided in Supplementary File 1 Table 3. 

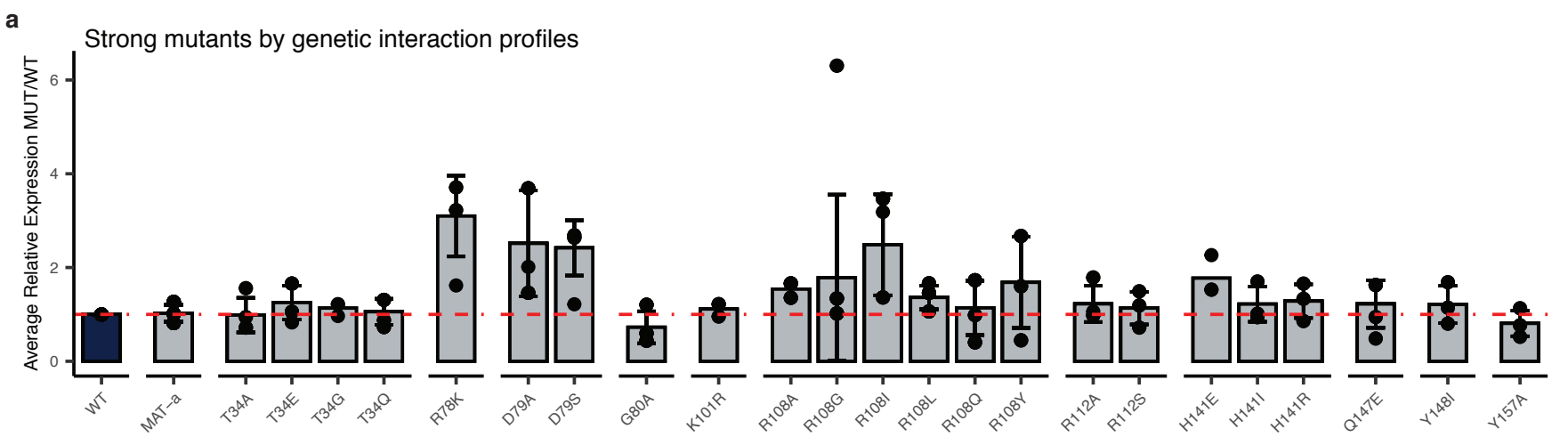

S. cerevisiae strain with genomically integrated Gsp1 point mutant

b

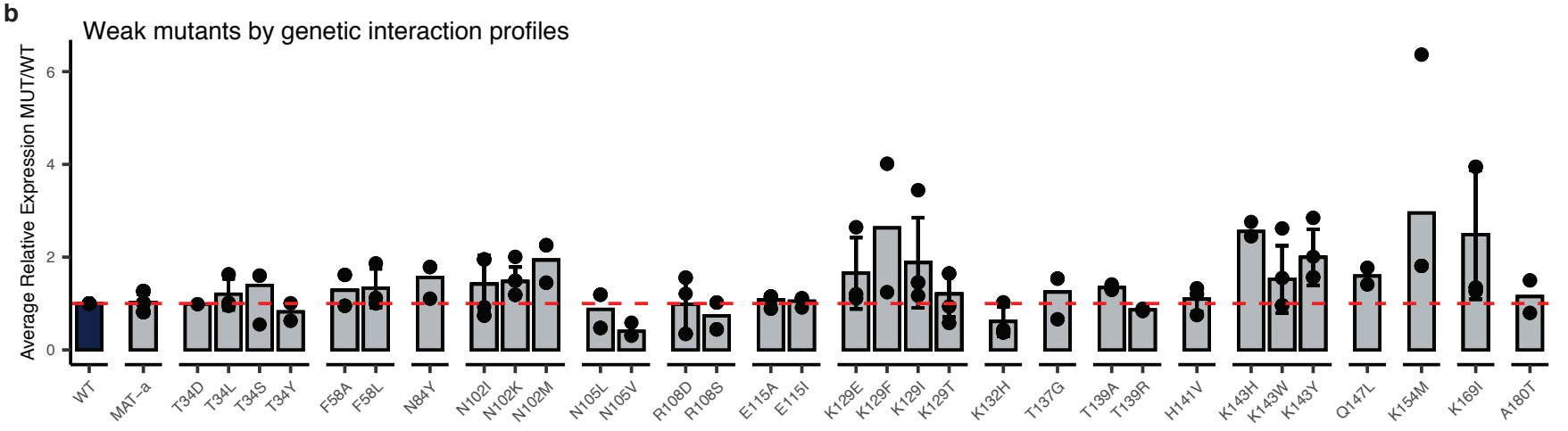

S. cerevisiae strain with genomically integrated Gsp1 point mutant

Fig. S2 Expression levels of endogenously expressed Gsp1 protein in S. cerevisiae strains with genomically integrated Gsp1 point mutations profiled by Western Blot. Expression levels are relative to the expression levels of wild-type Gsp1 protein. a, Expression data for strong mutants, defined as mutants with more than nine significant Gls. b, Expression data for weak mutants, defined as mutants with fewer than nine significant Gls. Bar heights indicate averages over 2 or more biological replicates (n) with error bars indicating one standard deviation for $n>=3$. Overlaid points indicate individual biological replicates (each an average over at least 12 technical replicates per biological replicate for wild-type and MAT- $\alpha$ strains, and between one and six technical replicates per biological replicate for mutant strains). Dashed red line indicates expression at the level of wild-type Gsp1 (fold change of 1). 
bioRxiv preprint doi: https://doi.org/10.1101/2020.01.04.893909; this version posted January 6, 2020. The copyright holder for this preprint (which was not certified by peer review) is the author/funder, who has granted bioRxiv a license to display the preprint in perpetuity. It is made available under aCC-BY-NC-ND 4.0 International license.

a

Genetic interaction (S-score)

S. cerevisiae E-MAP deletion library $(n=1444)$

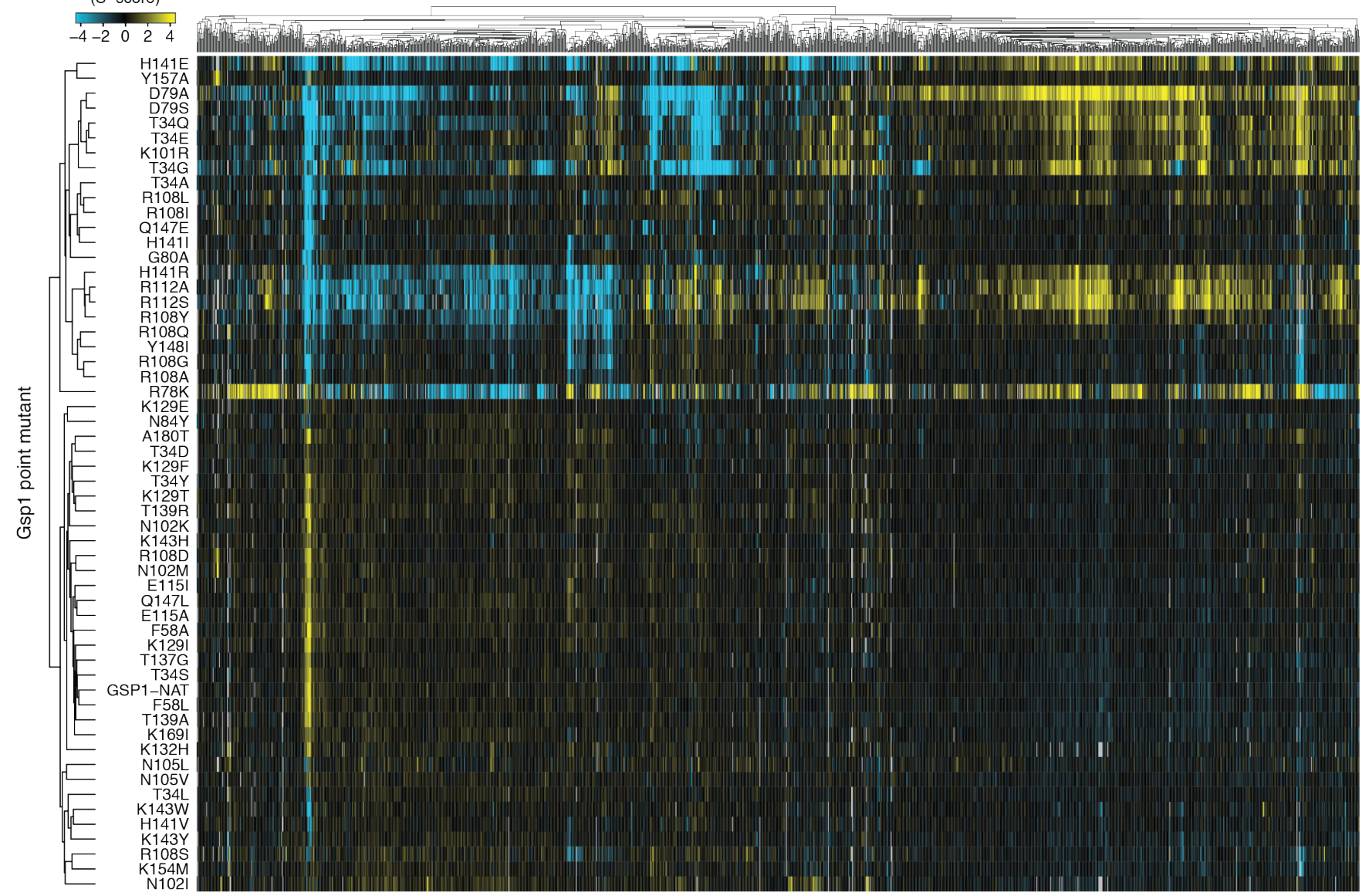

b

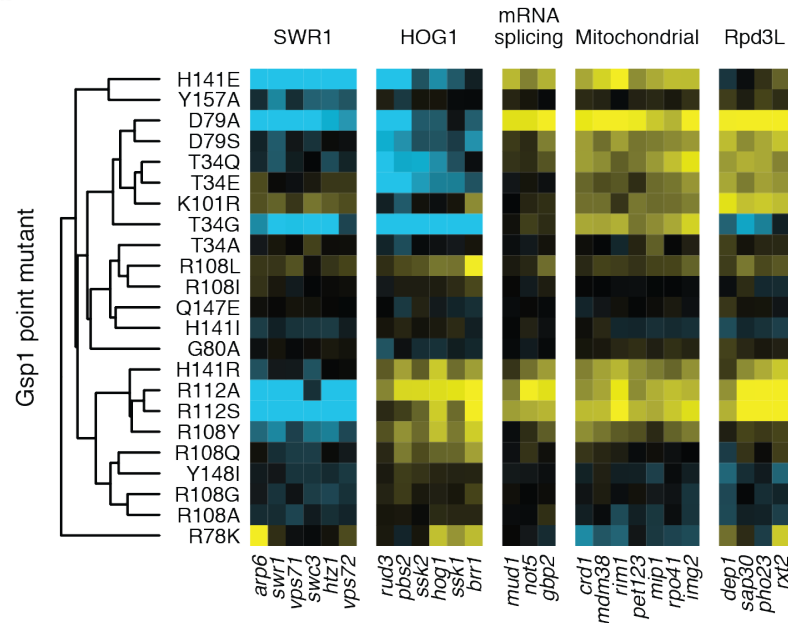

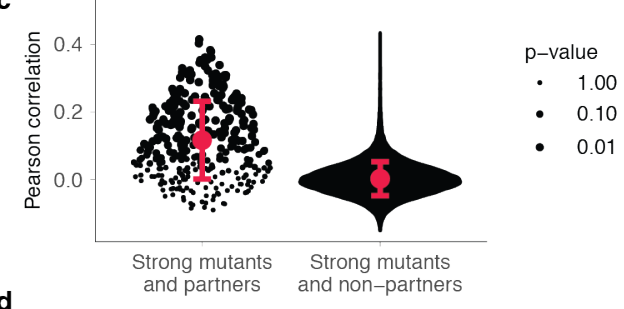

d

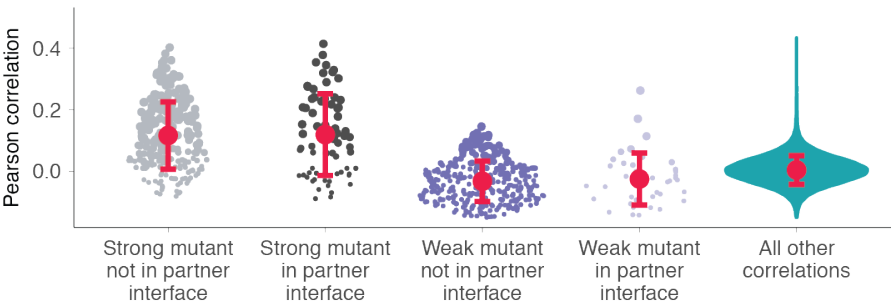

Fig. S3 Genetic interaction (GI) profiles of the 56 Gsp1 point mutations. a, Complete Gsp1 E-MAP profile. Negative S-score (blue) represents synthetic sick/lethal Gls, positive S-score (yellow) represents suppressive/epistatic Gls; neutral S-scores (no significant Gl) are shown in black. Mutants and genes are hierarchically clustered by Pearson correlation. Gsp1 mutants fall into two clusters: a cluster of 23 strong mutants with nine or more significant Gls (blue and yellow S-scores in the heatmap) and 23 weak mutants with fewer than nine significant Gls (mostly black S-scores in the heatmap). b, Gl profiles of Gsp1 mutants group $S$. cerevisiae genes by biological processes and complexes, such as the SWR1 complex, the Hog1 signaling pathway, mRNA splicing, mitochondrial proteins, and the Rpd3L histone deacetylase complex. c, Distributions of Pearson correlations between the Gl profiles of strong Gsp1 mutants and alleles of Gsp1 direct interaction partners with available co-complex crystal structures (left, Fig. 5a) and strong Gsp1 mutants and all other S. cerevisiae genes (right). d, Distributions of Pearson correlations between the GI profiles of Gsp1 interaction partners and strong and weak Gsp1 mutants if mutation is (black and light purple) or is not (gray and dark purple) in the interface with that partner. Teal violin plot on the right represents the distribution of all other Pearson correlations between Gsp1 mutants and $S$. cerevisiae genes. In $\mathrm{c}$ and $\mathrm{d}$, point size indicates the false discovery rate adjusted one-sided (positive) p-value of Pearson correlation. Red dots and bars indicate the mean and the upper and lower quartile, respectively. 
bioRxiv preprint doi: https://doi.org/10.1101/2020.01.04.893909; this version posted January 6, 2020. The copyright holder for this preprint (which was not certified by peer review) is the author/funder, who has granted bioRxiv a license to display the preprint in perpetuity. It is made available under aCC-BY-NC-ND 4.0 International license.

a

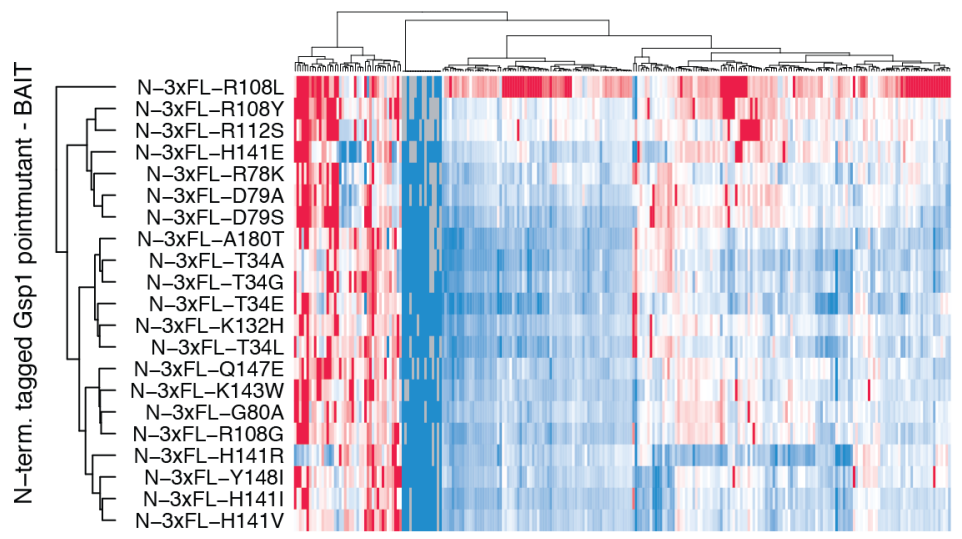

b

Gsp1 interaction partners - PREY protein from AP-MS ( $n=103)$

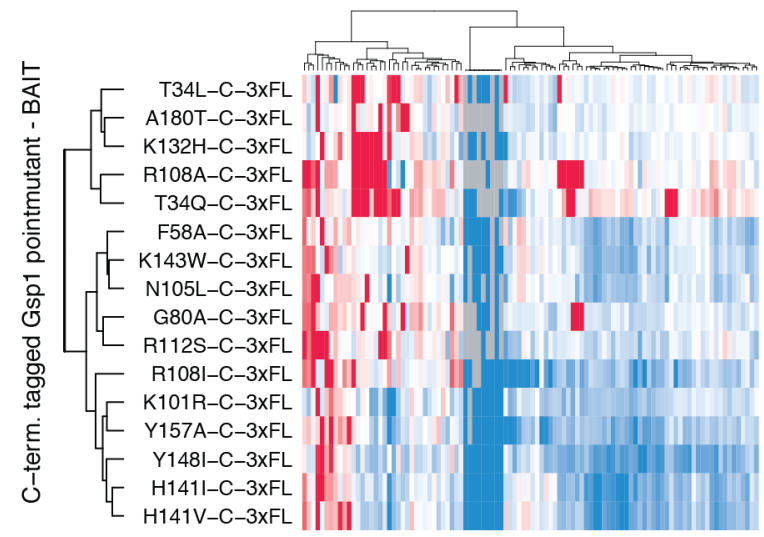

c

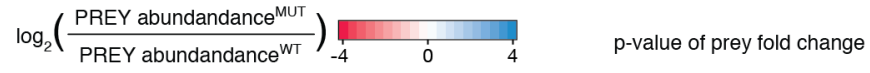

$<0.05 \bullet>0.05$

3xFLAG tag Gsp1 N-terminal C C-terminal

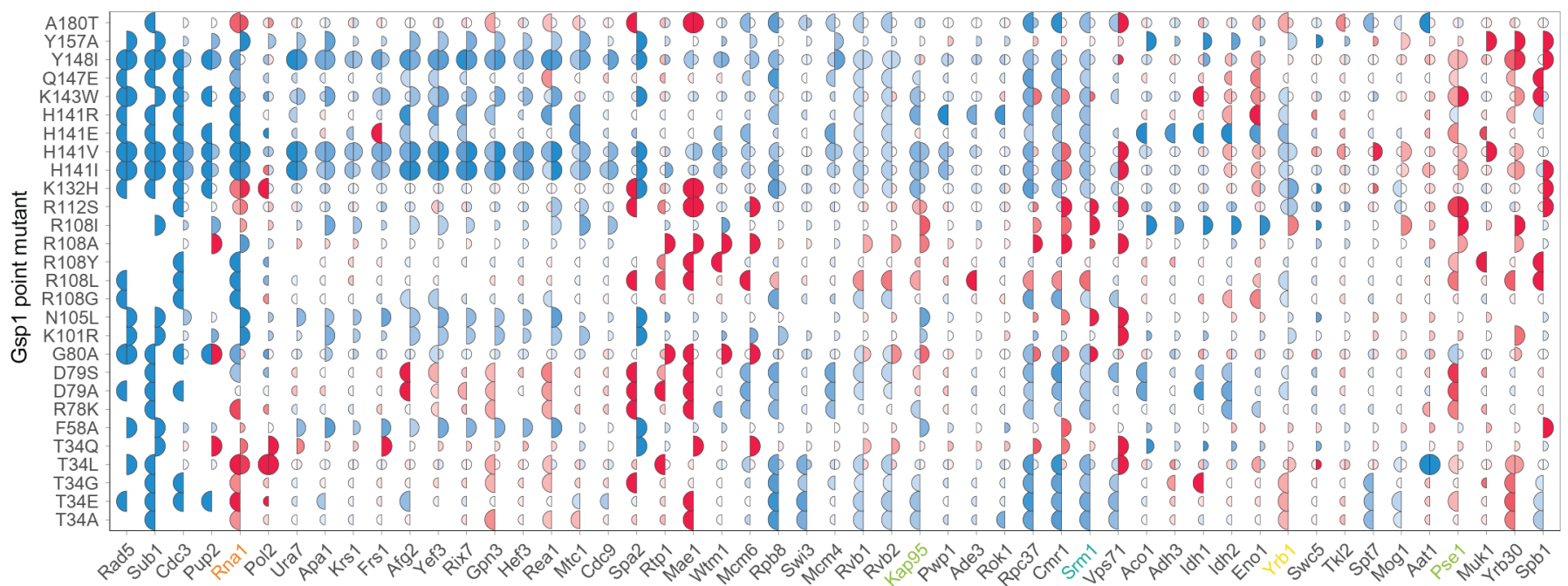

d

Intersection of Gsp1 interaction partners identified by AP-MS of Gsp1 strains with both tags $(n=51)$

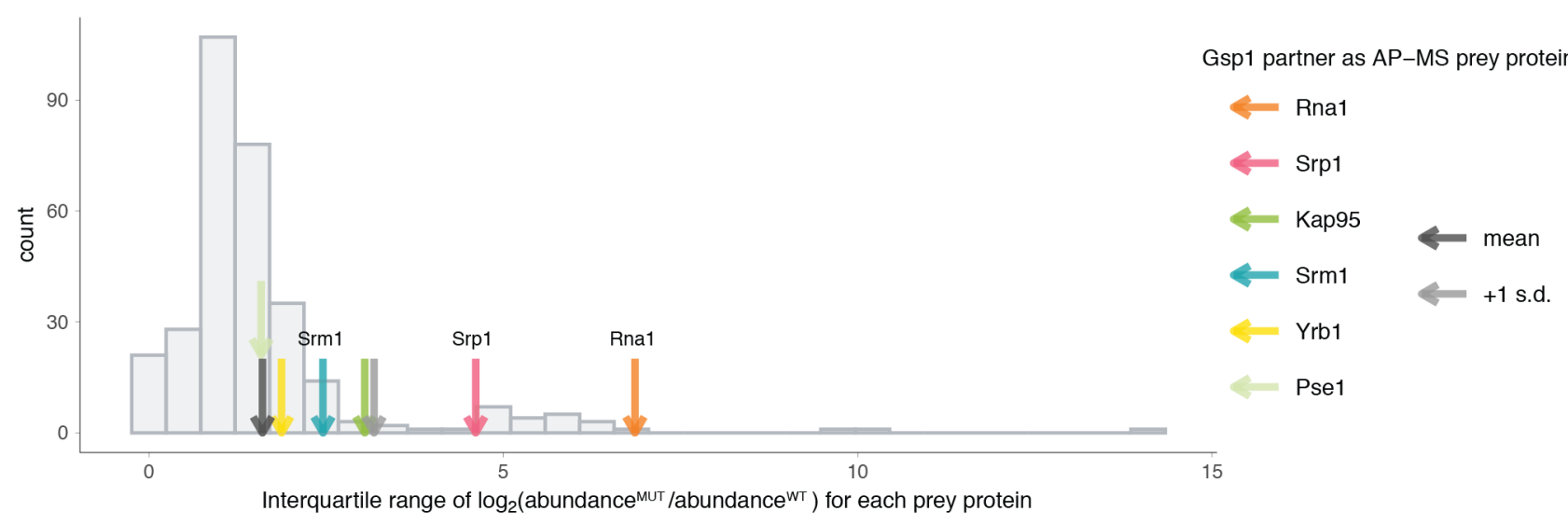

Fig. S4 Caption the the next page 
Fig. S4 Interface point mutations in Gsp1 rewire its physical interaction network. a, Amino- and b, -carboxy terminally 3xFLAG-tagged Gsp1 point mutants (rows) and prey proteins identified by AP-MS (columns) hierarchically clustered by the log ${ }^{-}$ transformed fold change in prey abundance pulled-down with either the mutant or wild-type Gsp1 with the corresponding 3xFLAG-tag $\left.\left(\log _{2}\left(\text { abundance }(P R E Y)^{\mathrm{MUT}} / \text { abundance(PREY }\right)^{\mathrm{WT}}\right)\right)$. c, Prey proteins pulled down by both amino- and carboxy-terminal tagged constructs. Left semi-circle represents an amino-terminal 3xFLAG-tagged Gsp1 point mutant, and right semi-circle represents carboxyterminal 3xFLAG-tagged Gsp1 point mutant. Semi-circle size is proportional to the significance of the $\log _{2}$-transformed fold change (false discovery rate adjusted p-value) of the prey abundance in pulled-down complexes with a Gsp1 mutant compared to complexes with the wild-type Gsp1. Overall we identified 316 high-confidence prey partner proteins, with the amino- and carboxy-terminally tagged Gsp1 mutants pulling down 264 and 103 preys, respectively, including 51 overlapping preys. The difference in preys identified by experiments with $\mathrm{N}$ - or C-terminal tags illustrates the sensitivity of the interaction network to perturbation of Gsp1. To account for possible tag effects, we always computed the fold change in prey abundance only relative to the wild-type protein with the corresponding tag. In a, b, and c, decreased abundance compared to pull-down with wild-type Gsp1 is annotated in red and increased abundance in blue.

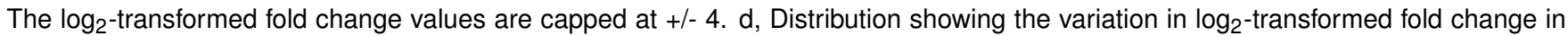
abundance of prey proteins shown in a and b, as defined by interquartile range (IQR) across mutants. Values for core partners shown as arrows (Rna1 orange, Srp1 pink, Kap95 green, Srm1 teal, Pse1 light green, Yrb1 yellow). Mean and +1 standard deviation of IQR values are highlighted with a dark gray and a light gray arrow, respectively. All IQR values are provided in Supplementary File 1 Table 5. 
bioRxiv preprint doi: https://doi.org/10.1101/2020.01.04.893909; this version posted January 6, 2020. The copyright holder for this preprint (which was not certified by peer review) is the author/funder, who has granted bioRxiv a license to display the preprint in perpetuity. It is made available under aCC-BY-NC-ND 4.0 International license.

a

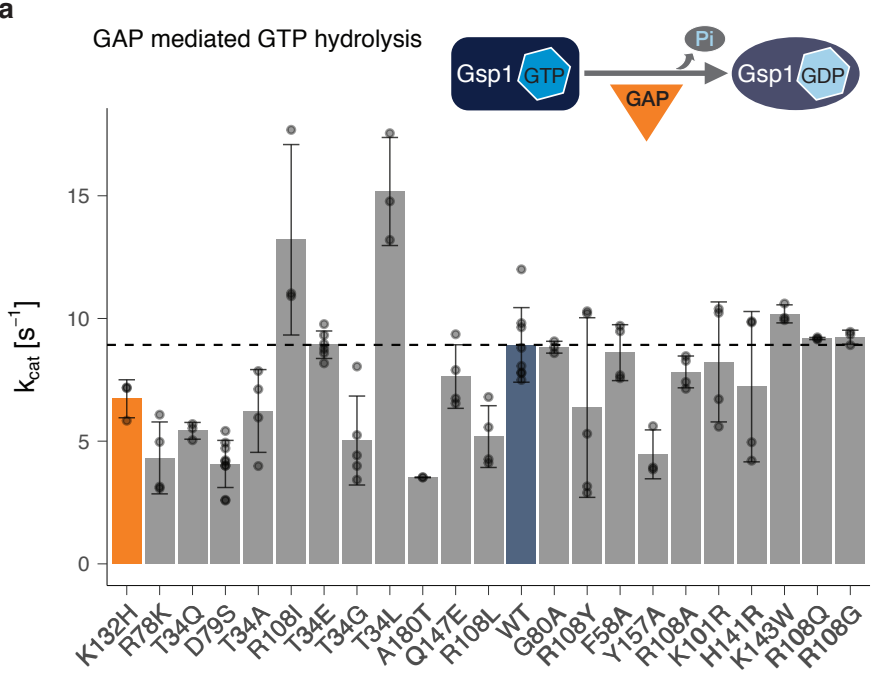

b

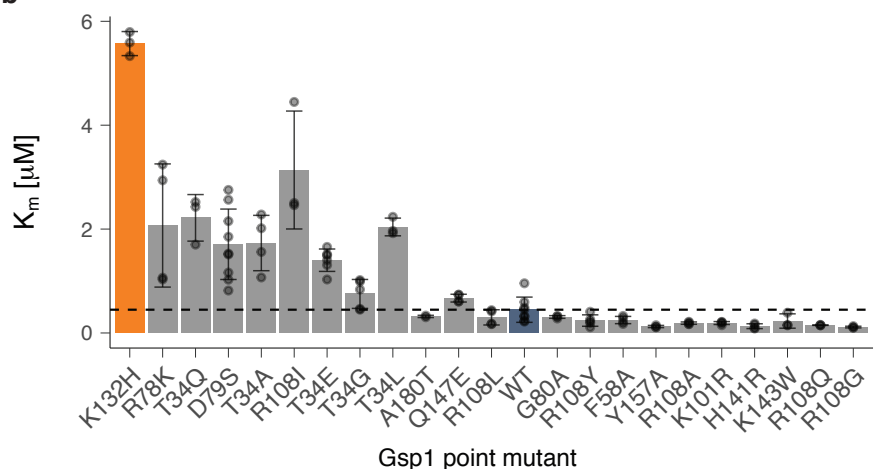

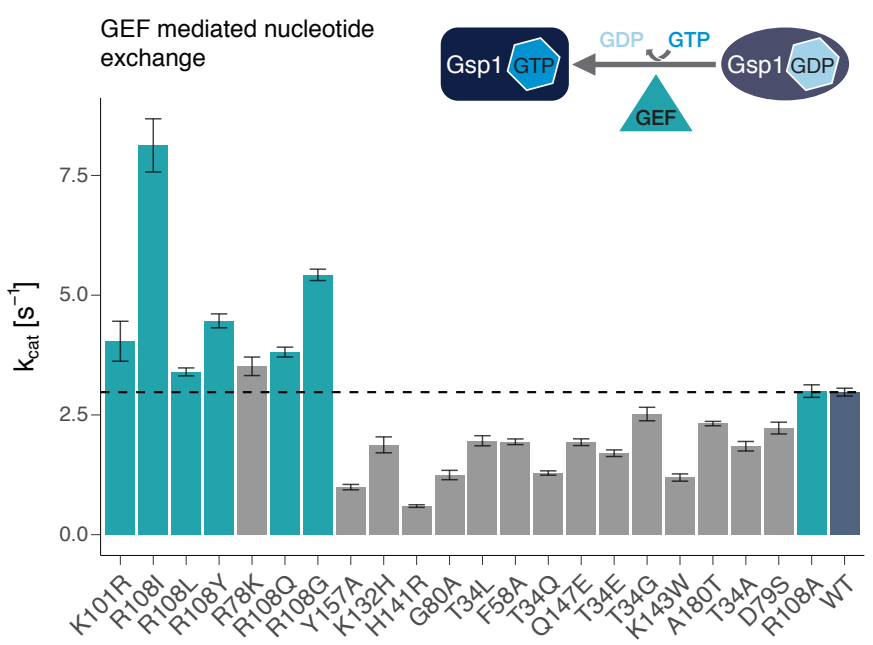

d

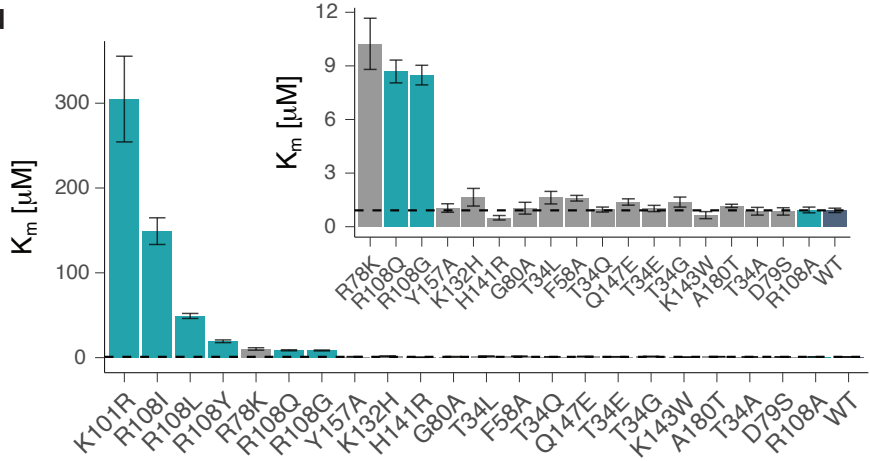

Gsp1 point mutant

WT Gsp1 $\square$ in GEF interface $\square$ in GAP interface $\square$ not in either interface

e

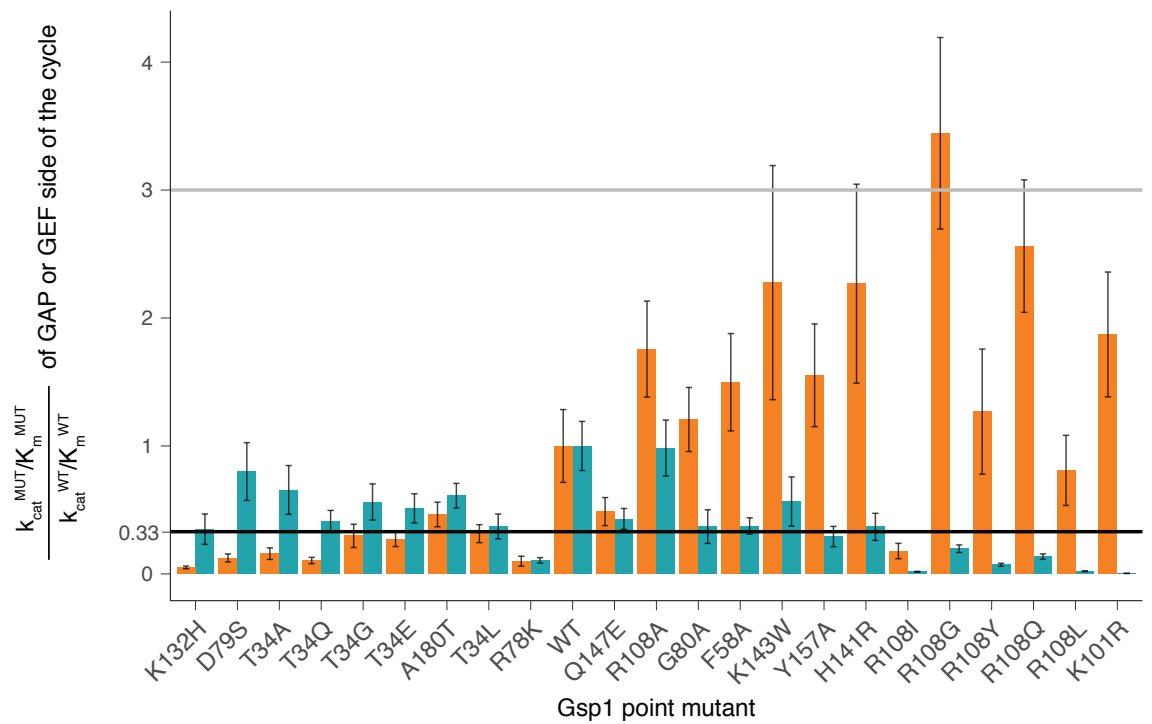

GAP mediated GTP hydrolysis

GEF mediated nucleotide exchange

Fig. S5 Caption on the next page 
Fig. S5 Effect of Gsp1 point mutations on the in vitro efficiency of GAP-mediated GTP hydrolysis and GEF-mediated nucleotide exchange. $\mathbf{a}, \mathrm{k}_{\mathrm{cat}}$ and $\mathbf{b}, \mathrm{K}_{\mathrm{m}}$ values of GAP-mediated GTP hydrolysis of wild-type and point mutant Gsp1. Error bars represent the standard deviation of the $\mathrm{K}_{\mathrm{cat}}$ and the $\mathrm{K}_{\mathrm{m}}$ parameters from the integrated Michaelis-Menten fit for $\mathrm{n} 3$ replicates (except for A180T which has two replicates). $\mathbf{c}, \mathrm{k}_{\mathrm{cat}}$ and $\mathbf{d}, \mathrm{K}_{\mathrm{m}}$ of GEF-mediated nucleotide exchange of wild-type and point mutant Gsp1. Inset shows the $\mathrm{K}_{\mathrm{m}}$ barplot for all but the four mutants with the highest $\mathrm{K}_{\mathrm{m}}$. Error bars represent the value plus/minus the standard error of the MichaelisMenten fit to data from $n 17$ measurements at different substrate concentrations. $\mathbf{a}, \mathbf{b}, \mathbf{c}$, $\mathbf{d}$, Dotted lines indicate the wild-type values. Dark blue bar denotes the wild-type Gsp1, and orange and teal bars highlight the residues that are in the interface with the GAP and GEF, respectively. e, Comparison of relative change in catalytic efficiencies of the GAP-mediated GTP hydrolysis (orange bars) and GEF-mediated nucleotide exchange (teal bars) defined as $k_{\text {cat }}{ }^{M U T} / K_{m}{ }^{M U T} / k_{c a t}{ }^{W T} / K_{m}{ }^{W T}$. Gray line indicates a three-fold increase compared to wild type, black line indicates a three-fold decrease compared to wild type. Error bars represent the added standard error of the mean (for GAP) or standard error of the fit (for GEF) values of the mutant and the wild-type efficiency $\left(k_{\text {cat }} / K_{m}\right)$ values. 
bioRxiv preprint doi: https://doi.org/10.1101/2020.01.04.893909; this version posted January 6, 2020. The copyright holder for this preprint (which was not certified by peer review) is the author/funder, who has granted bioRxiv a license to display the preprint in perpetuity. It is made available under aCC-BY-NC-ND 4.0 International license.

${ }^{31}$ P NMR spectrum of WT Gsp1 loaded with GTP

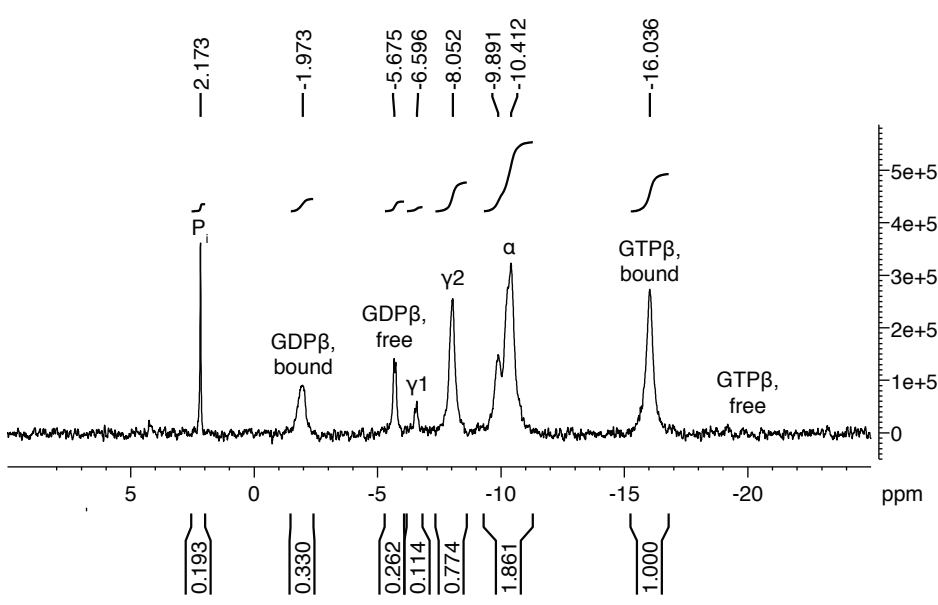

C

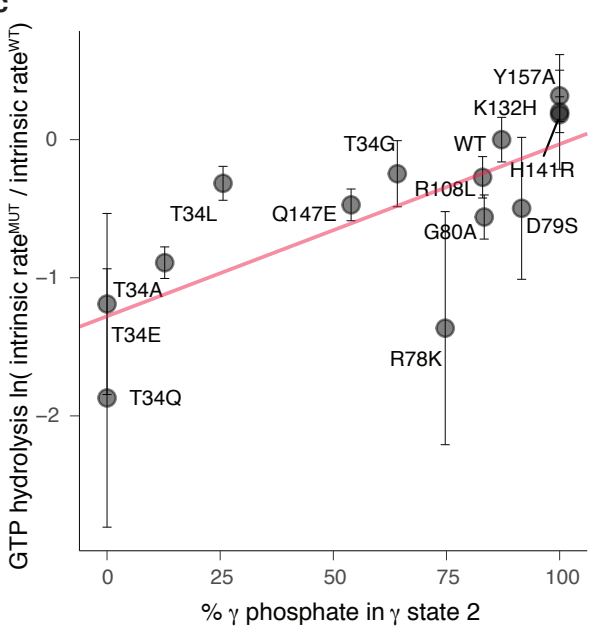

d b Intrinsic GTP hydrolysis rate

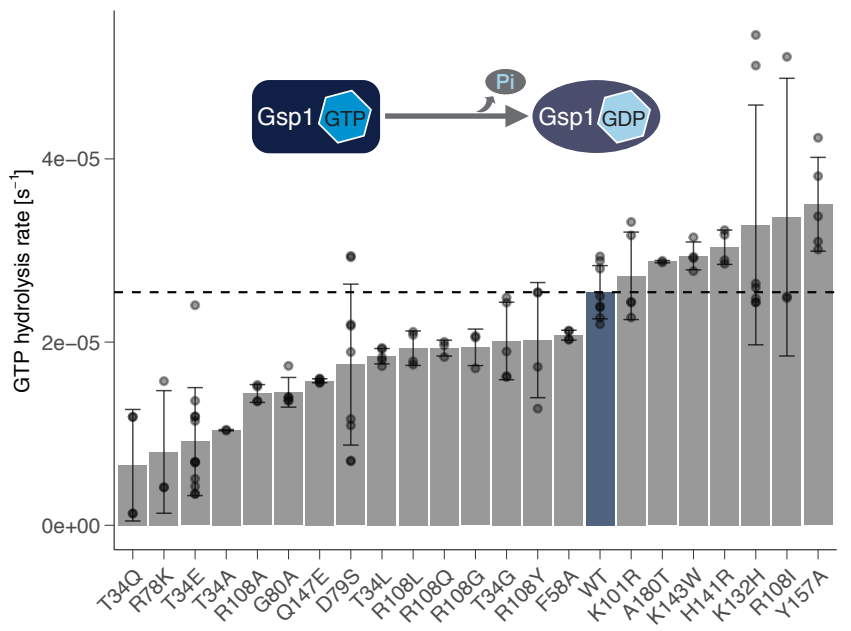

Gsp1 point mutant

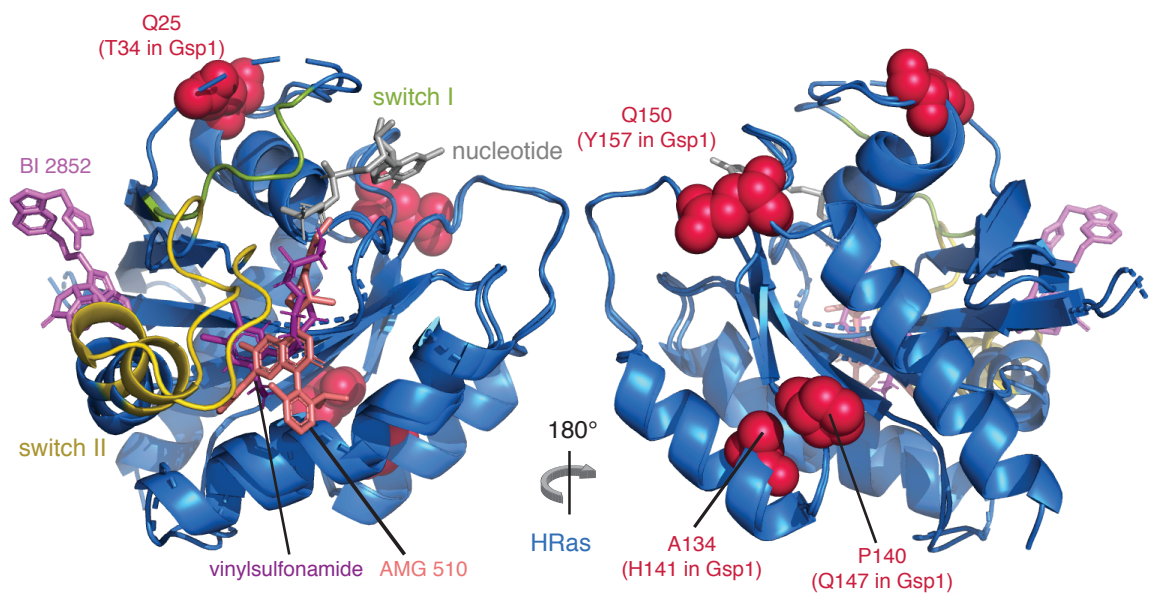

Fig. S6 Gsp1 interface mutations act allosterically to modulate the rate of GTP hydrolysis. a, Annotated $1 D^{31} P$ NMR spectrum of wild-type Gsp1 loaded with GTP. Peak areas are computed over intervals shown and normalized to the GTP $\beta$ bound (GTP $\beta$ bound) peak. The peaks from left to right correspond to: free phosphate (Pi), $\beta$ phosphate of GDP bound to Gsp1 (GDP $\beta$ bound), $\beta$ phosphate of free (unbound) GDP (GDP $\beta$ free), $\gamma$ phosphate of GTP bound to Gsp1 in conformation 1 ( $\gamma 1$ ), $\gamma$ phosphate of GTP bound to Gsp1 in conformation $2(\gamma 2), \alpha$ phosphate of bound or unbound GDP or GTP, $\beta$ phosphate of GTP bound to Gsp1 (GTP $\beta$ bound), $\beta$ phosphate of free (unbound) GTP (GTP $\beta$ free). b, Rate of intrinsic GTP hydrolysis of wild-type Gsp1 and mutants. Dotted line indicates wild-type value. Error bars represent the standard deviations from $n 3$ replicates (except for A180T which has two replicates). c, Percent population in $\gamma 2$ state plotted against the relative rate of intrinsic GTP hydrolysis represented as a natural logarithm of the ratio of the rate for the mutant over the rate of the wild type. The pink line is a linear fit. Error bars represent the standard deviation from $n$ replicates of intrinsic GTP hydrolysis measurements (except for A180T which has two replicates). d, Structures of HRas (in cartoon representation, blue) bound to allosteric inhibitors shown in stick representation: BI 2852 (PDB ID: 6gj8, light violet), vinylsulfonamide (PDB ID: 4m1w, dark violet), and AMG 510 (PDB ID: 6oim, deepsalmon). Switch I and switch II regions of HRas are in green and yellow, respectively. Human HRas residues corresponding to Gsp1 allosteric sites (identified from the sequence alignment between Gsp1 and human HRas) are represented as pink spheres. The corresponding Gsp1 residues are in parentheses. 
bioRxiv preprint doi: https://doi.org/10.1101/2020.01.04.893909; this version posted January 6, 2020. The copyright holder for this preprint (which was not certified by peer review) is the author/funder, who has granted bioRxiv a license to display the preprint in perpetuity. It is made available under aCC-BY-NC-ND 4.0 International license.
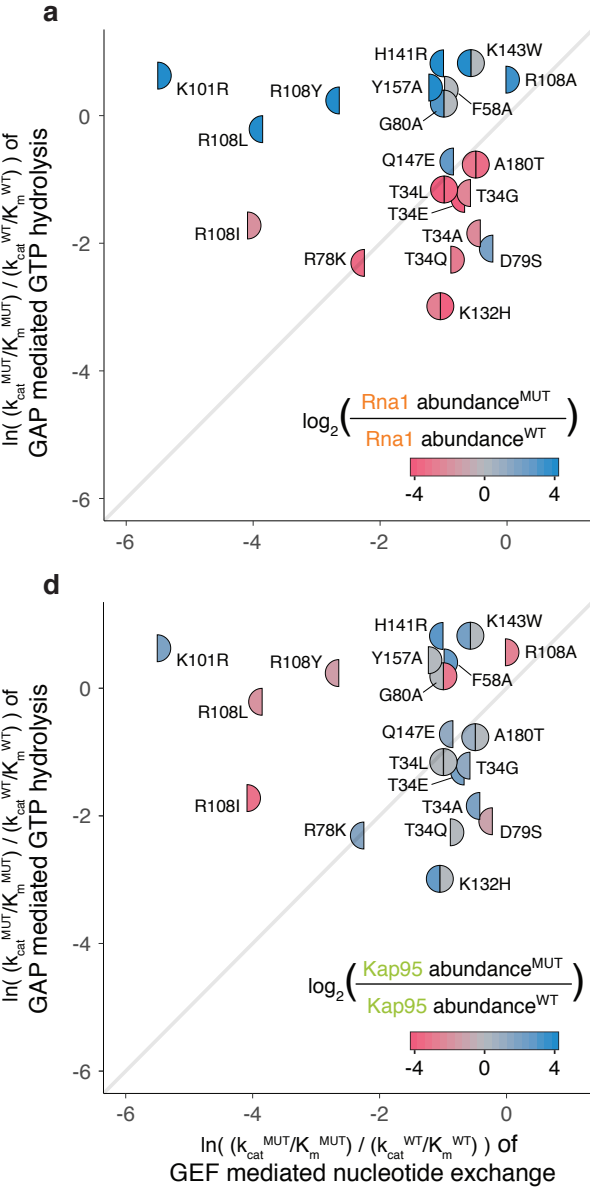

b

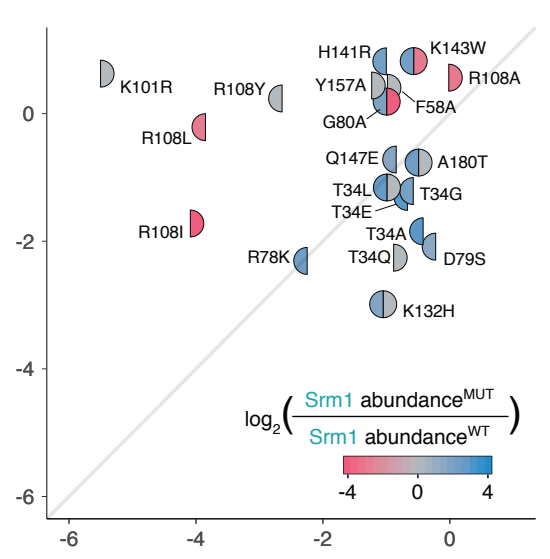

e

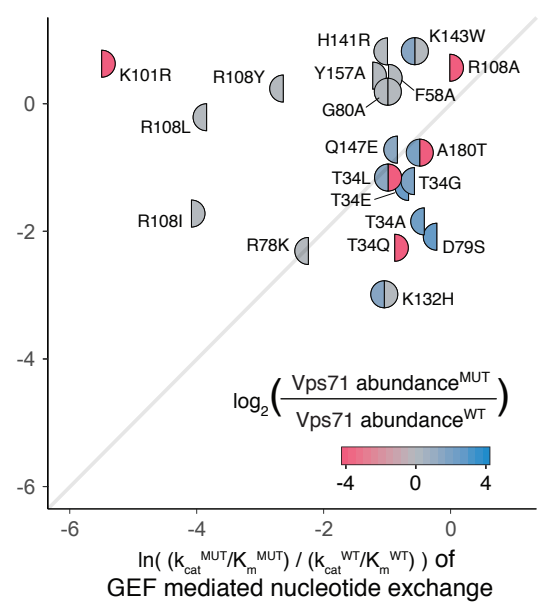

c

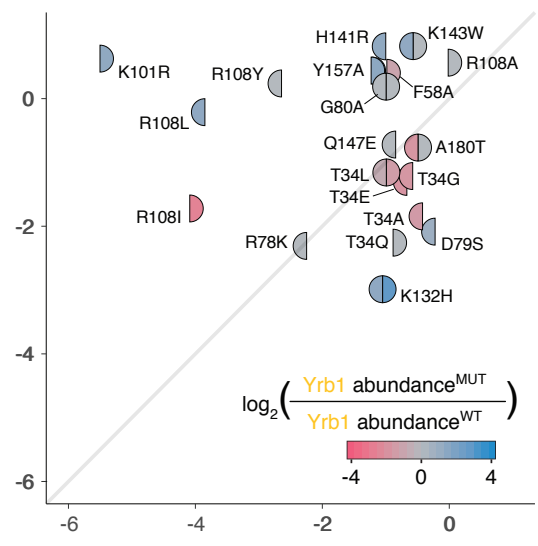

f

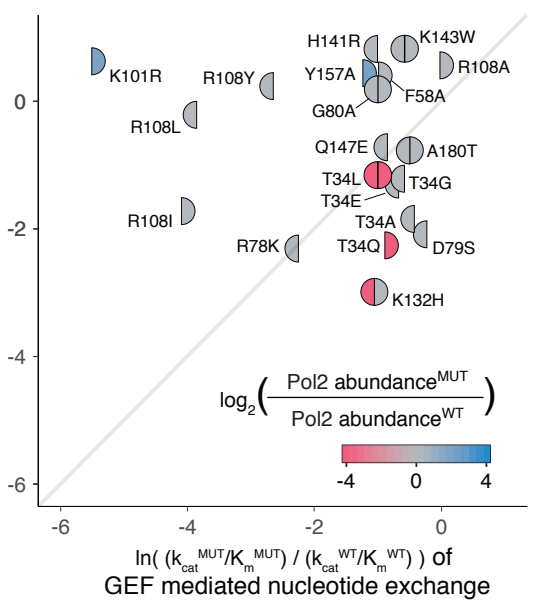

3xFLAG tag Gsp1 N-terminal C C-terminal

Fig. 57 Changes in relative prey protein abundance $\log _{2}$ (prey abundance ${ }^{\mathrm{MUT}} /$ prey abundance ${ }^{\mathrm{WT}}$ ) for each Gsp1 point mutant compared to the wild type with corresponding 3xFLAG tag from the AP-MS proteomics experiment, overlaid onto the effects of each mutation on relative in vitro efficiencies of GAP-mediated GTP hydrolysis and GEF-mediated nucleotide exchange. Relative GAP-mediated hydrolysis and GEF-mediated exchange efficiencies are defined as $\ln \left(\mathrm{k}_{\mathrm{cat}}{ }^{\mathrm{MUT}} / \mathrm{K}_{\mathrm{m}}{ }^{\mathrm{MUT}} / \mathrm{k}_{\mathrm{cat}}{ }^{\mathrm{WT}} / \mathrm{K}_{\mathrm{m}}{ }^{\mathrm{WT}}\right)$. Decreased prey abundance from AP-MS in pulled-down complexes with a mutant Gsp1 compared to complexes with the wild-type Gsp1 is represented in red and increased abundance in blue. The $\log _{2}$ (PREY abundance ${ }^{\mathrm{MUT}} / \mathrm{PREY}$ abundance ${ }^{\mathrm{WT}}$ ) values are capped at $+/-4$. Left semi-circle represents an amino-terminal 3xFLAG-tagged Gsp1 point mutant, and right semi-circle represents carboxy-terminal 3xFLAG-tagged Gsp1 point mutant, relative to wild-type Gsp1 with the corresponding tag. Prey proteins: a, Rna1 (GAP); b, Srm1 (GEF); c, Yrb1; d, Kap95; e, Vps71; and f, Pol2. 
bioRxiv preprint doi: https://doi.org/10.1101/2020.01.04.893909; this version posted January 6, 2020. The copyright holder for this preprint (which was not certified by peer review) is the author/funder, who has granted bioRxiv a license to display the preprint in perpetuity. It is made available under aCC-BY-NC-ND 4.0 International license.

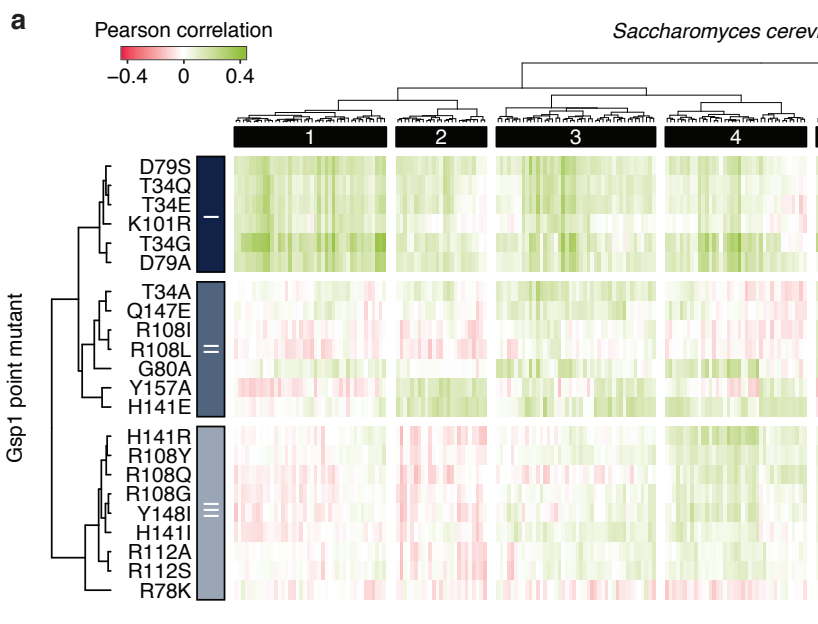

b

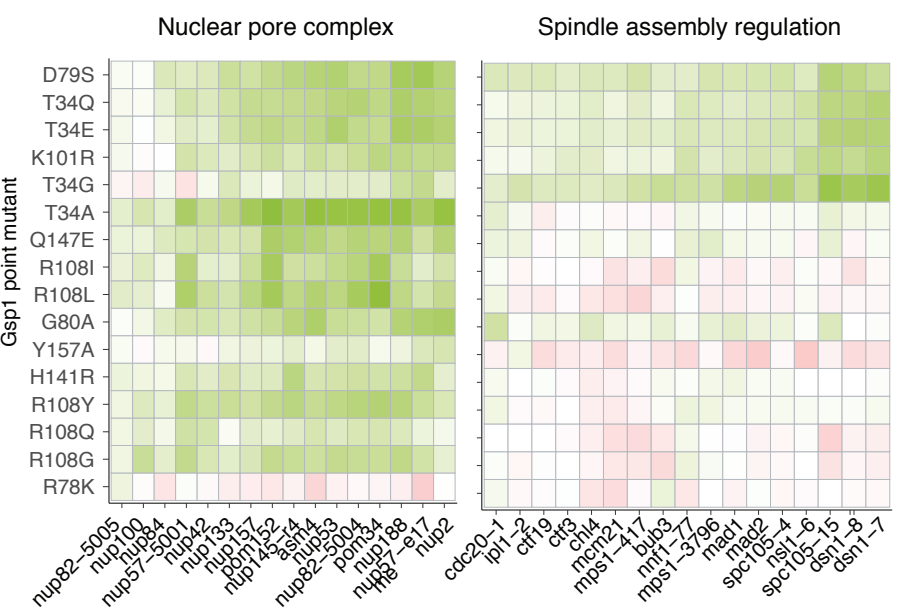
tRNA modification

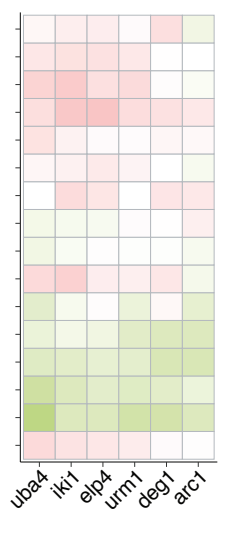

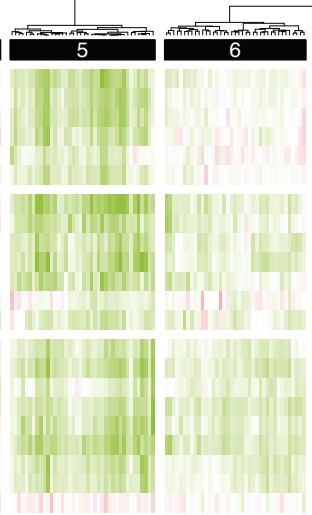

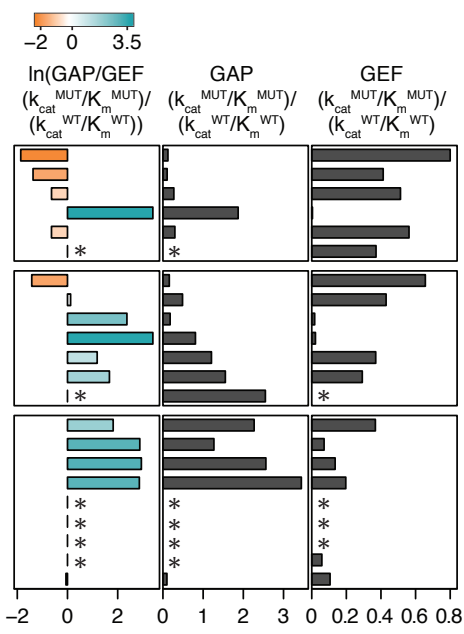

e
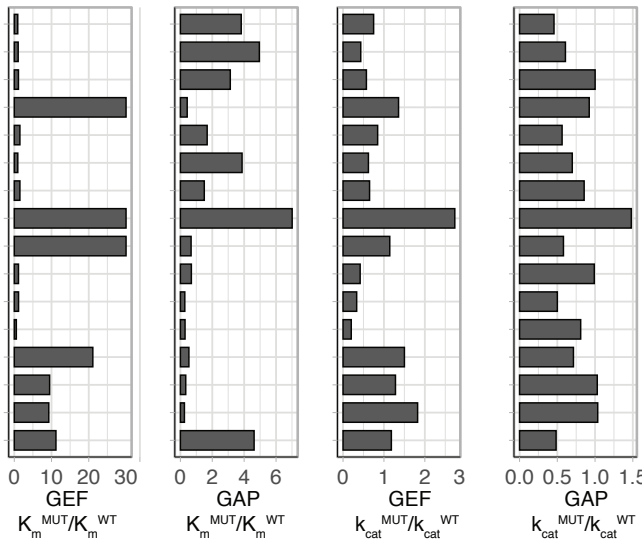

Fig. S8 Pearson correlation coefficients between GI profiles of Gsp1 point mutants and S. cerevisiae genes recapitulate the approximate ordering of Gsp1 mutants by the effects of point mutations on the GAP-mediated GTP hydrolysis and the GEFmediated nucleotide exchange. a, The matrix of Pearson correlations between the Gl profiles of the 22 Gsp1 point mutants and $S$. cerevisiae genes is shown using the cluster ordering based on p-value from Fig. 4b compared to GAP-mediated GTP hydrolysis and GEF-mediated nucleotide exchange efficiencies $\left(\mathrm{k}_{\mathrm{cat}} / \mathrm{K}_{\mathrm{m}}\right)$ as indicated. Positive (green) and negative (red) Pearson correlations are capped at +/- 0.4. The 278 S. cerevisiae genes all have at least two significant correlations with a Gsp1 mutant (see Methods). A star next to the relative kinetic values indicates that no kinetics data were collected for that mutant. b-d, Heatmaps of gene sets shown in Fig. 4c colored by Pearson correlations. e, Relative $\mathrm{k}_{\text {cat }}$ and $\mathrm{K}_{\mathrm{m}}$ values of GEF-mediated nucleotide exchange and GAP-mediated GTP hydrolysis for Gsp1 mutants compared to wild type. 
bioRxiv preprint doi: https://doi.org/10.1101/2020 01.04 893909; this version posted January 6, 2020. The copyright holder for this preprint (which was not certified by peer review) is the author/funder, who has granted bioRxiv a license to display the preprint in perpetuity. It is made available under aCC-BY-NC-ND 4.0 International license.

a

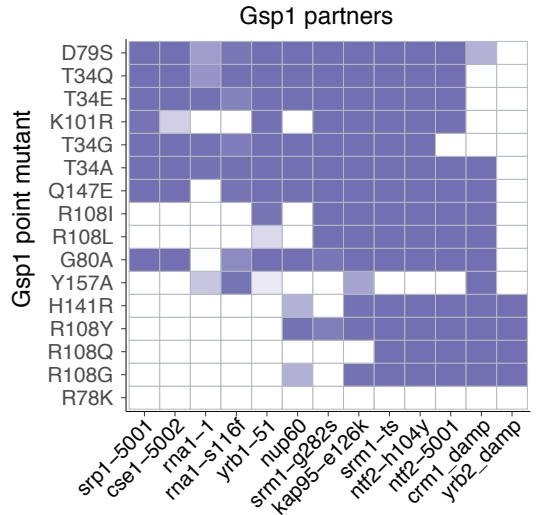

b

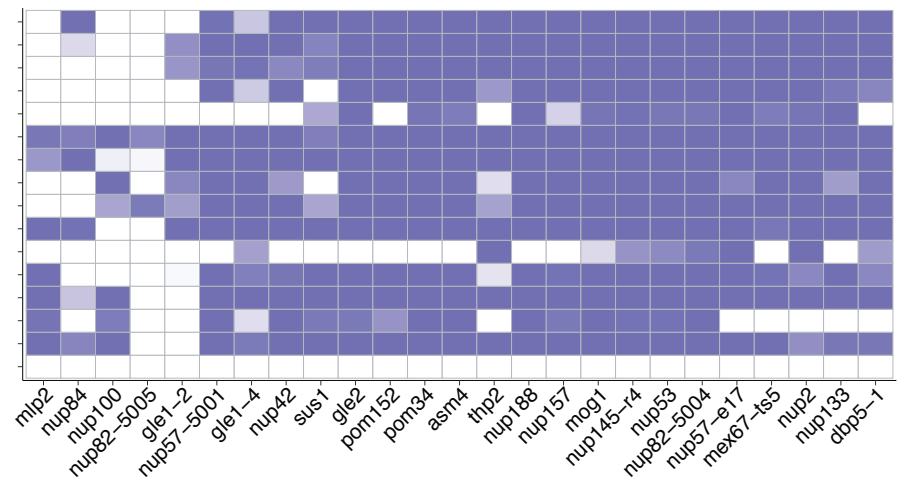

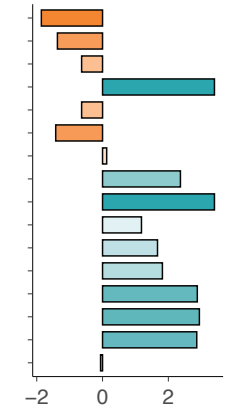

In(GAP/GEF

$\left.\left(\mathrm{k}_{\text {cat }}{ }^{\mathrm{MUT}} / \mathrm{K}_{\mathrm{m}} \mathrm{MUT}^{\mathrm{N}}\right) /\left(\mathrm{k}_{\text {cat }}{ }^{\mathrm{WT}} / \mathrm{K}_{\mathrm{m}}{ }^{\mathrm{WT}}\right)\right)$

c
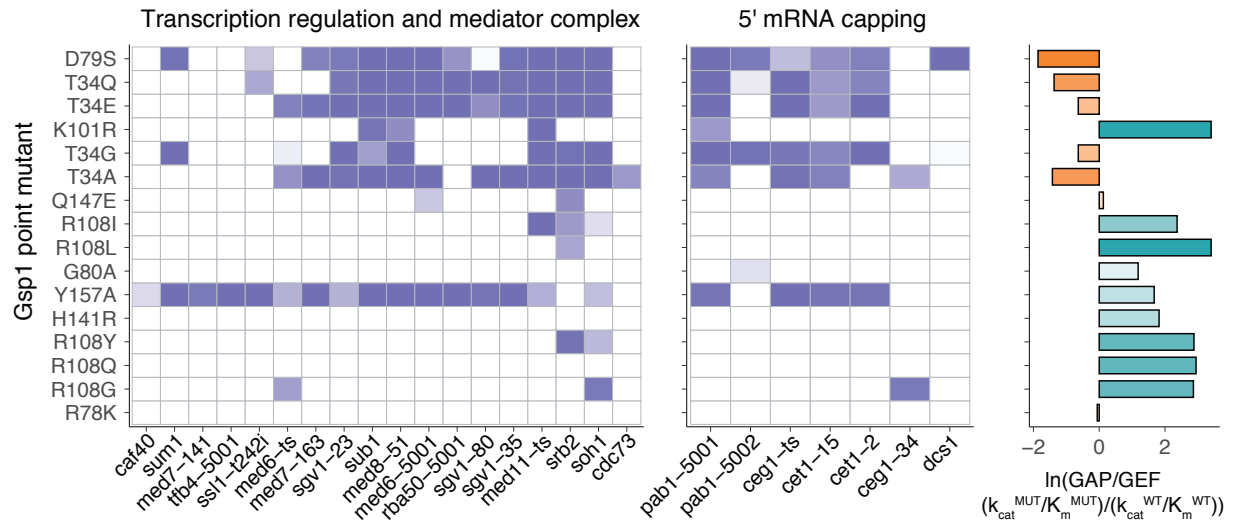

$\underset{\left(k^{M U T} / K^{M U T}\right) /}{\ln (\mathrm{GAP} / \mathrm{GEF}}$

$\left.\left(\mathrm{K}_{\text {cat }} \mathrm{WWT}_{\mathrm{T}} / \mathrm{K}_{\mathrm{m}}^{\mathrm{WT}}\right)\right)$

p-value of GI

Pearson correlations
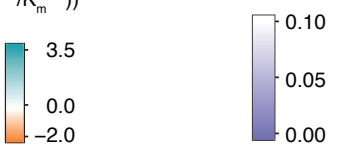

d
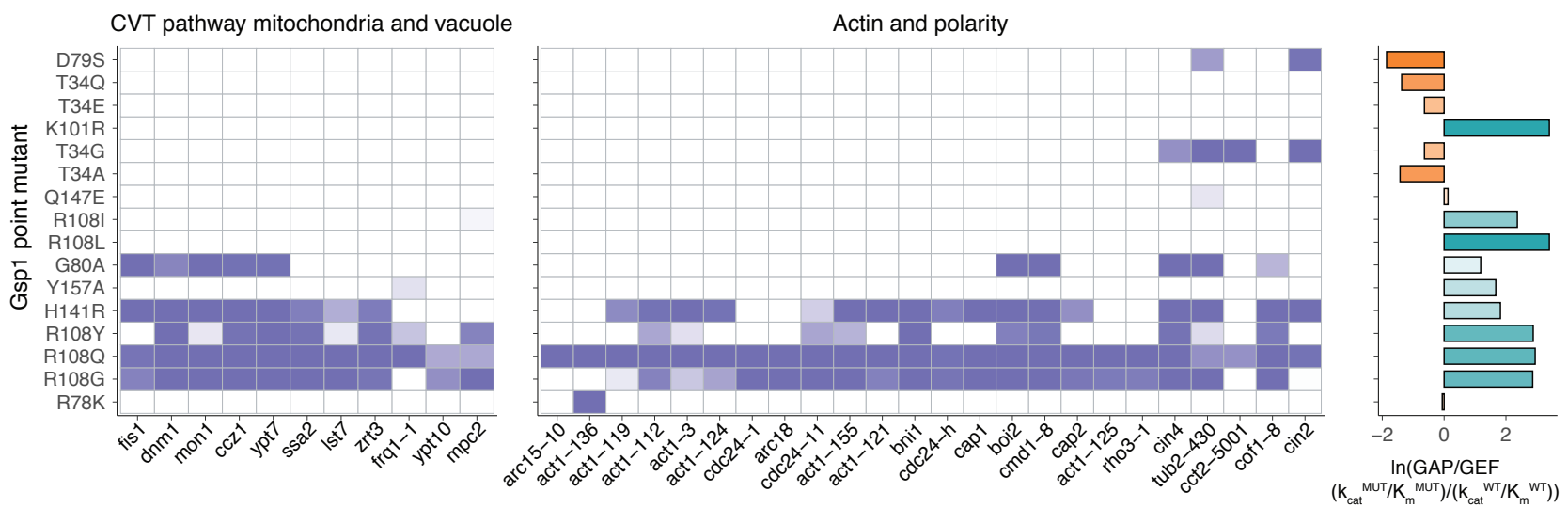

e

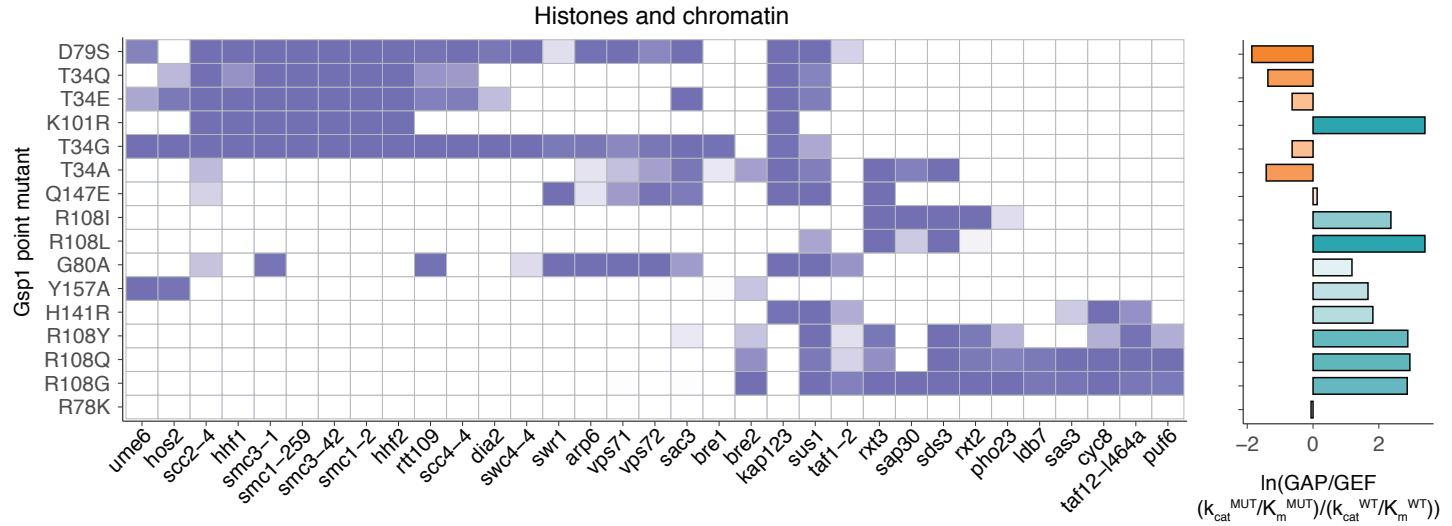

Fig. S9 Caption on the next page 
bioRxiv preprint doi: https://doi.org/10.1101/2020.01.04.893909; this version posted January 6, 2020. The copyright holder for this preprint (which was not certified by peer review) is the author/funder, who has granted bioRxiv a license to display the preprint in perpetuity. It is made available under aCC-BY-NC-ND 4.0 International license.

Fig. S9 Sets of S. cerevisiae genes grouped by biological functions correlate either with all Gsp1 mutants that perturb the balance of the GTPase cycle, or correlate better with mutants that perturb either one or the other side of the GTPase cycle. Heatmaps of the false discovery rate adjusted one-sided (positive) p-values of the Pearson correlations between 22 strong Gsp1 point mutants and knock-outs or knock-downs of $S$. cerevisiae genes from the CellMap. The p-value is represented as a white to purple range, with purple being most significant. Genes are organized in gene sets based on their biological function (Methods). a, Gsp1 point mutants and alleles of Gsp1 binding partners with available co-complex X-ray crystal structures. b, Gsp1 point mutants and S. cerevisiae genes involved in nuclear transport of RNA and proteins. c, Gsp1 point mutants and S. cerevisiae genes involved in transcription regulation or 5' mRNA capping. d, Gsp1 point mutants and S. cerevisiae genes involved in the cytoplasm-to-vacuole targeting (CVT) pathway and cell polarity. e, Gsp1 point mutants and S. cerevisiae genes involved in histone modifications and chromatin. 